\title{
WINGS: A WIde-field Nearby Galaxy-cluster Survey
}

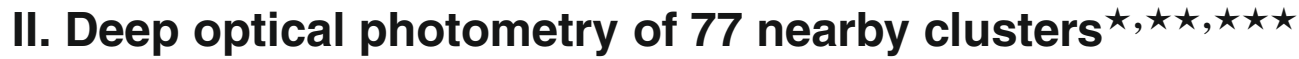

\author{
J. Varela ${ }^{1,2}$, M. D’Onofrio ${ }^{3}$, C. Marmo ${ }^{4}$, G. Fasano ${ }^{1}$, D. Bettoni ${ }^{1}$, A. Cava ${ }^{1,5}$, W. J. Couch ${ }^{6}$, \\ A. Dressler ${ }^{7}$, P. Kjærgaard ${ }^{8}$, M. Moles $^{2}$, E. Pignatelli ${ }^{1}$, B. M. Poggianti ${ }^{1}$, and T. Valentinuzzi ${ }^{3}$ \\ 1 INAF - Padova Astronomical Observatory, Vicolo Osservatorio 5, 35122 Padova, Italy \\ e-mail: jesus.varela.lopez@gmail.com \\ 2 Instituto de Astrofísica de Andalucía (C.S.I.C.) Apartado 3004, 18080 Granada, Spain \\ 3 Astronomy Department, University of Padova, Vicolo Osservatorio 2, 35122 Padova, Italy \\ 4 UMR 8148 IDES Interactions et Dynamique des Environnements de Surface Université, Paris-Sud, 91405 Orsay Cedex, France \\ 5 Instituto de Astrofísica de Canarias, c/ Vía Láctea s/n, La Laguna, Spain \\ ${ }^{6}$ Centre for Astrophysics and Supercomputing, Swinburne University of Technology, Hawthorn, VIC 3122, Australia \\ 7 Observatories of the Carnegie Institution of Washington, Pasadena, CA 91101, USA \\ 8 The Niels Bohr Institute, Juliane Maries Vej 30, 2100 Copenhagen, Denmark
}

Received 31 March 2008 / Accepted 11 December 2008

\section{ABSTRACT}

Context. This is the second paper of a series devoted to the WIde Field Nearby Galaxy-cluster Survey (WINGS). WINGS is a long term project which is gathering wide-field, multi-band imaging and spectroscopy of galaxies in a complete sample of 77 X-ray selected, nearby clusters $(0.04<z<0.07)$ located far from the galactic plane $\left(|b| \geq 20^{\circ}\right)$. The main goal of this project is to establish a local reference for evolutionary studies of galaxies and galaxy clusters.

Aims. This paper presents the optical $(B, V)$ photometric catalogs of the WINGS sample and describes the procedures followed to construct them. We have paid special care to correctly treat the large extended galaxies (which includes the brightest cluster galaxies) and the reduction of the influence of the bright halos of very bright stars.

Methods. We have constructed photometric catalogs based on wide-field images in $B$ and $V$ bands using SExtractor. Photometry has been performed on images in which large galaxies and halos of bright stars were removed after modeling them with elliptical isophotes.

Results. We publish deep optical photometric catalogs (90\% complete at $V \sim 21.7$, which translates to $\sim M_{V}^{*}+6$ at mean redshift), giving positions, geometrical parameters, and several total and aperture magnitudes for all the objects detected. For each field we have produced three catalogs containing galaxies, stars and objects of "unknown" classification $(\sim 16 \%)$. From simulations we found that the uncertainty of our photometry is quite dependent of the light profile of the objects with stars having the most robust photometry and de Vaucouleurs profiles showing higher uncertainties and also an additional bias of $\sim-0.2^{\mathrm{m}}$.

The star/galaxy classification of the bright objects $(V<20)$ was checked visually making negligible the fraction of misclassified objects. For fainter objects, we found that simulations do not provide reliable estimates of the possible misclassification and therefore we have compared our data with that from deep counts of galaxies and star counts from models of our Galaxy. Both sets turned out to be consistent with our data within $\sim 5 \%$ (in the ratio galaxies/total) up to $V \sim 24$.

Finally, we remark that the application of our special procedure to remove large halos improves the photometry of the large galaxies in our sample with respect to the use of blind automatic procedures and increases $(\sim 16 \%)$ the detection rate of objects projected onto them.

Key words. galaxies: clusters: general - catalogs

\section{Introduction}

Clusters of galaxies are privileged systems to study, among others, two basic problems. On one hand, they (or a substantial part of them at least) are the largest gravitationally bound

* Based on observations taken at the Issac Newton Telescope (2.5 mINT) sited at Roque de los Muchachos (La Palma, Spain), and the MPG/ESO-2.2 m Telescope sited at La Silla (Chile).

$\star \star$ Appendices are only available in electronic form at http://www. aanda.org

$\star \star \star$ Catalog is only available in electronic form at the CDS via anonymous ftp to cdsarc.u-strasbg. fr $(130.79 .128 .5)$ or via http://cdsweb.u-strasbg.fr/cgi-bin/qcat?J/A+A/497/667 structures and as such, physical entities whose properties must be explained by cosmological theories. On the other hand, clusters are made of hundreds or even thousands of galaxies in high density regions whose properties can be studied and straightforwardly compared and their evolution analyzed.

Clusters of galaxies were first detected as marked overdensities in the projected number of galaxies. Even if today it has been recognized that galaxies represent only a small fraction of the total mass of the clusters, they still are a fundamental tool to study cluster properties since they are usually much more easily detected and measured than the X-ray emitting intracluster gas or the more evasive non-baryonic component. 
Clusters of galaxies also have been widely used to study the evolution of galaxies in dense environments. Some of the first clear evidence of evolution of the galaxies came from the study of the populations of galaxies in clusters made by Butcher $\&$ Oemler (1978). They found that the fraction of blue galaxies was higher in clusters at $z \gtrsim 0.4$ than in nearby clusters, which they interpreted as the result of the aging of spiral galaxies after losing their gas supply and, therefore, diminishing their star formation rates. In parallel, Dressler (1980) showed that the central, denser parts of clusters are mainly populated by early type galaxies. Since then, several works have discussed the idea that morphological content in clusters of galaxies changes along the Hubble time (Dressler et al. 1997; Fasano et al. 2000; van Dokkum et al. 2000; Lubin et al. 2002; Postman et al. 2005).

Two are the main factors that make clusters useful to study the evolution of galaxies. First, galaxies in clusters can be considered as being at the same distance since usually this distance is much greater than the linear dimensions of the clusters. Therefore, knowing the redshift of a small subsample of the cluster is enough to (statistically) know the distance (once background correction it is allowed for) of hundreds or even thousands of galaxies. Of course, this highly increases the statistics when analyzing the properties of the galaxies. Second, clusters of galaxies can be detected up to high redshifts using different techniques that are sensitive to local enhancements of the galaxy density or through the $\mathrm{X}$ ray emission from the intracluster medium.

Using the first all sky survey (The National Geographic Society-Palomar Observatory Sky Survey, Abell 1959) the first systematic catalogs of clusters of galaxies were constructed (Abell 1957; Zwicky et al. 1963). However, it was only in the 1970 s when clusters of galaxies started to be used as laboratories to study the properties of the galaxies they contain and how their evolution is affected by the environment (Gunn \& Gott 1972; Oemler 1974). From this point on, the interest in clusters of galaxies at always higher redshift continuously grew and nowadays, specially thanks to the Hubble Space Telescope, clusters at $z \gtrsim 1$ have been studied in detail (e.g. RDCS J1252 2927 at $z=1.235$; Postman et al. 2005) and using different techniques protoclusters have been detected at $z>2$ (e.g. Steidel et al. 2000; Kurk et al. 2004).

To produce a correct interpretation of the observations at different redshifts a good knowledge of the cosmic variance of the properties of clusters and galaxies at each redshift is needed, to ensure that the changes observed with distance are statistically significant. Paradoxically, as we discussed in Fasano et al. (2006, hereafter Paper I), there is a relative lack of knowledge of the properties of the clusters of galaxies in the local Universe at $z \sim 0$ and the comparison of higher redshift clusters is always done with single clusters such as Virgo, Coma or Fornax. Indeed, for more than 20 years the most complete study of galaxies in nearby clusters of galaxies has been that by Dressler (1980). Dressler's work was based on data extracted from photographic plates. Several programs have been set up to continue that work with modern tools and techniques. Each of them addresses a particular aspect or problem and, therefore, we still lack a general local reference for evolutionary studies. Thus, the ESO Nearby Abell Cluster Survey (Katgert et al. 1996; Biviano et al. 1997, ENACS) includes spectroscopy of galaxies in a number of clusters but the imaging is not as deep as that of Dressler's work. The Las Campanas/ATT Rich Cluster Survey (O'Hely et al. 1998; Pimbblet et al. 2001) is deeper but includes only 20 clusters. In recent years, large sky surveys as the $2 \mathrm{dF}$ (De Propris et al. 2002) and, particularly, the SDSS (Goto et al. 2002; Bahcall et al. 2003;
Miller et al. 2005) have been sources for the compilation and analysis of large samples of clusters of galaxies. However, while the $2 \mathrm{dF}$ is only spectroscopic the SDSS, which covers a very large area, is not deep enough to study the faintest part of the luminosity function. More recently, the NOAO Fundamental Plane Survey (Smith et al. 2004) started to fill this lack of data, however its main goal is not the study of the evolution of galaxies and clusters but the large scale velocity fields using the Fundamental Plane ${ }^{1}$.

In this context, the WIde-field Nearby Galaxy-clusters Survey (Paper I) has been presented with the specific goal of sampling the properties of clusters and galaxies in clusters in the local Universe. This means to establish both the average and the variance of the properties of clusters and of the galaxies they contain.

Briefly, WINGS is a long term multiwavelength project based on deep optical $(B, V)$ wide field images $\left(\sim 35^{\prime} \times 35^{\prime}\right)$ of 77 fields centered on nearby clusters of galaxies selected from three X-ray flux limited samples compiled from ROSAT AllSky Survey data (BCS, Ebeling et al. 1996; eBCS, Ebeling et al. 1998; XBACs, Ebeling et al. 2000). The selected redshift range $(0.04<z<0.07)$ was set to balance a wide linear field of view $(\sim 1.4 \mathrm{Mpc} \times 1.4 \mathrm{Mpc}$ at $z \sim 0.04)$ and a high spatial resolution $\left(1.34 \mathrm{kpc} /{ }^{\prime \prime}\right.$ at $\left.z \sim 0.07\right)$. To reduce the effects of galactic extinction in our analysis, only clusters located far from the galactic equator $\left(|b| \geq 20^{\circ}\right)$ were kept. The observations were carried out using the wide field cameras of two telescopes: the $2.5 \mathrm{~m}$ Isaac Newton Telescope (WFC@INT) and the MPG/ESO-2.2 m telescope (WFI@ESO). The optical data were complemented with spectroscopic follow up of a subsample of 48 clusters (Cava et al. 2008) using WYFFOS@WHT ( $\lambda$ range $=3800-7000 \AA$, $\lambda$ resolution $=3 \AA$ ) and $2 \mathrm{dF} @ \mathrm{AAT}(\lambda$ range $=3600-8000 \AA$, $\lambda$ resolution $=6 \AA$ ). Also, we obtained $J$ and $K$ imaging of 32 WINGS clusters with WFCAM@UKIRT (Valentinuzzi et al. 2008) and we are collecting $\mathrm{U} / \mathrm{H}_{\alpha}$ band imaging of WINGS clusters with wide-field cameras at different telescopes (INT, LBT, Bok), that will be used to analyse the stellar masses and of star formation.

Paper I started the series devoted to the analysis of the optical data within the so called WINGS-OPT subproject. It presented the criteria followed to construct the initial sample of clusters and described the optical observations ( $B$ and $V$ bands) and their photometric and astrometric quality.

This is the second paper in the series regarding the optical photometry of the WINGS project and it is devoted to the release of the catalogs containing the basic photometric parameters of all the objects found in the fields of 77 nearby clusters of galaxies. Also, it describes the procedures that were followed to construct the catalogs (Sect. 2). The process includes a preliminary treatment of the images (Sect. 2.1) that has allowed us to improve the photometry of many galaxies, especially the largest ones. In Sect. 3, catalogs are presented and their overall quality is checked including their photometric quality (Sect. 3.2.1), completeness and star/galaxy separation (Sect. 3.2.3). A final summary will be found in Sect. 4.

Throughout this paper we will use a cosmological model with parameters: $H_{0}=75 \mathrm{~km} \mathrm{~s}^{-1} \mathrm{Mpc}^{-1}, \Omega_{\mathrm{M}}=0.3$ and $\Omega_{\Lambda}=0.7^{2}$.

\footnotetext{
1 A more detailed comparison from the spectroscopic point of view will be made in Cava et al. (2008).

$2 \mathrm{Be}$ aware that in Paper I we used instead $H_{0}=70 \mathrm{~km} \mathrm{~s}^{-1} \mathrm{Mpc}^{-1}$, however this change doesn't modify the results showed there
} 

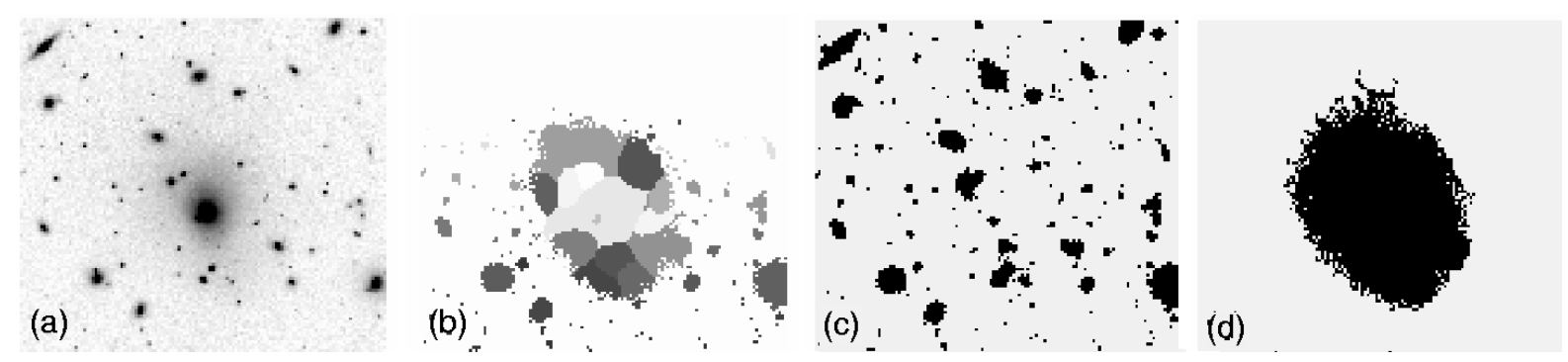

Fig. 1. Example of the problems of SExtractor to produce the correct segmentation of the extended galaxies. From left to right: a) BCG of cluster A193 in the $V$ band image; b) segmentation performed by SExtractor without subtracting the galaxy; each color represents those pixels assigned by SExtractor to different objects; c) segmentation of the remaining objects after subtraction of the BCG; d) final segmentation of the BCG.

\section{Detection, basic photometry and star-galaxy classification}

The whole process of source detection, computation of their basic photometric parameters and star-galaxy classification was performed using SExtractor (Bertin \& Arnouts 1996). SExtractor is a very efficient tool to find sources in large format images. Among its characteristics the most interesting are its ability to separate blended sources and to estimate the probability of an object of being a star or an extended source. However, both processes need to be fine-tuned by input parameters that are fixed for each single image. To improve the final outcome of SExtractor a preliminary treatment of the images was done before running the source detection program as well as a final interactive checking of the star-galaxy classification, as described in the following.

\subsection{Preliminary image treatment}

In images like ours in which the ranges of size and brightness are quite large, it is impossible in practice to find a single set of values of SExtractor's input parameters that could work adequately for all the objects. The extreme situation is when a large galaxy is contaminated by a number of small projected sources as is the case of the brightest central galaxies (BCGs) of our sample of clusters. This issue is illustrated in Fig. 1 with the BCG of the cluster A193 (panel (a)). The segmentation ${ }^{3}$ of the central galaxy and the objects projected onto it is shown in panel (b). Clearly, the program has erroneously assigned pixels of the large galaxy to the small projected objects producing poor photometry of the large galaxy as well as of the objects projected onto it. Something similar happens with very bright stars whose extended halos affect the photometry of the nearby objects.

To minimize the effect of such large halos in the photometry of close objects as well as to improve the photometry of the extended galaxies themselves we have developed a custom-made procedure. It consists of first the modeling and removal of these halos before running SExtractor on the image. This improves the photometry of the small projected objects. In a second step, an image is constructed containing only the extended galaxies and the photometry is performed on it with SExtractor. In this last image the pixels of the projected small objects are replaced by the values of the models and the rest of the pixels are left unchanged. This replacement reduces the contamination of the projected objects in the photometry of the large galaxies. An additional advantage of the procedure is that the removal of the

\footnotetext{
3 The segmentation is the way in which a program of source detection assigns pixels to each object.
}

extended halos also improves the determination of the global background map.

In the following we give a brief description of the process that is explained in more detail in Appendix A.

The process starts with the computation of a first background map which is subtracted from the image. Then, the problematic objects (galaxies and stars with extended halos) are located. For each of these objects, a mask of the projected objects is constructed and elliptical isophotes are fit ${ }^{4}$. The resulting fit is used to construct a model of the halo that is afterwards subtracted from the original image (i.e. the image before the background subtraction). After doing that with all the selected objects, the resulting image without the large halos is used to refine the background map as well as the masks of the smaller objects. With the new background map and masks the process is repeated to improve the final results. It is found that one iteration is enough to achieve sufficiently accurate photometry.

The last step is the construction of a complementary image containing only the previously removed galaxies (of course, the bright stars are also avoided in this image). It is important to note that the photometry of the large galaxies is not performed on the models but rather using the original pixels. The models are used only those regions occupied by projected galaxies or by interchip regions.

At the end of the whole process, which is run in $V$ band as well as in $B$ band, the output is two background subtracted images in each band. One of the images contains all the objects except the largest ones and the other one only the extended galaxies that were removed from the first image.

The improvement of the procedure is illustrated in the last two panels of Fig. 1 in which we show the segmentations of the projected objects (panel (c)) and of the BCG (panel (d)) after applying our procedure.

\subsection{Detection and basic photometry}

To construct the photometric catalogs, the detection of sources and the basic photometry relies on SExtractor and more specifically on the modified version SExtractor 2.3 .2 by Morrison $^{5}$ following the changes initially made by Holwerda for SExtractor v.2.2.2. These versions have the advantage of computing additional parameters related to the light distribution in

\footnotetext{
4 To achieve a successful fit a preliminary "masking" procedure is needed of the objects that appear projected onto the halos. See Appendix A for more details.

5 http://www.cfht.hawaii.edu/ morrison/home/

SExtractor.html
} 
the objects such as the concentration, the contrast and the asymmetry (Abraham et al. 1994, 1996).

The detection of sources, the determination of their positions and geometrical parameters as well as the star/galaxy classification was done on the $V$ band image. Since we had images from two different cameras with different pixel scales, instead of setting a single detection threshold per pixel for both cameras we opted for setting the same detection threshold per square arcsec. The advantage of this approach is that with similar observational conditions the limiting surface brightness is independent of the pixel size. The disadvantage is that in the images with lower detection threshold per pixel (in our case the WFI@ESO) the rate of spurious detections at low brightness is higher. However, since we were interested in the bright part of the luminosity function, where the galaxies of the cluster outnumber those of the background, it was desirable to perform the photometry of the galaxies within similar surface brightness limits. In order to balance photometric depth (i.e. detection of low surface brightness galaxies) with a low rate of spurious detections, the detection threshold was set to $4.5 \sigma_{\mathrm{bg}} / \operatorname{arcsec}^{2}$ ( $\sigma_{\mathrm{bg}}$ is the standard deviation of the background signal) which corresponds to $1.5 \sigma_{\mathrm{bg}} / \mathrm{pixel}$ for the WFC@INT and 1.07 $\sigma_{\mathrm{bg}}$ / pixel for the WFI@ESO. Given the typical values of $\sigma_{\mathrm{bg}}$ found in our images these limits translate in a detection limit of $\mu_{\text {Threshold }}(V) \sim 25.7 \mathrm{mag} / \operatorname{arcsec}^{2}$. As a comparison, at the same signal to noise level the images from the $\operatorname{SDSS}^{6}$ in $g$ band reach $\mu_{\text {Threshold }}(g)<25.2 \mathrm{mag} / \operatorname{arcsec}^{2}$ while those in $r$ band reach $\mu_{\text {Threshold }}(r)<24.7 \mathrm{mag} / \operatorname{arcsec}^{2}$.

SExtractor was run twice with the $B$ band images using both the single-image and the dual-image modes ${ }^{7}$. The catalog resulting from running SExtractor in single-image mode allowed us to reduce the number of spurious detection because only objects with detections in both bands (separated by less than 1'.67 = 5 pixel (WFC@INT)=7 pixel (WFI@ESO)) were kept. However, to obtain correct color indexes of the galaxies we performed a second run of SExtractor on the $B$ band image using the dual-image mode in which the $V$ band image was set as the detection image. This procedure ensured that the measurements of each galaxy in both filters were done in the same regions.

SExtractor was run without using weighted images because preliminary tests showed that the photometry was more robust when not using weighting images ${ }^{8}$. As a consequence, photometric errors computed by SExtractor are unrealistically small. The estimation of these errors is explained in Sect. 3.2.1.

To avoid the influence of the interchip regions, the pixels in these regions were set to zero and the objects falling within a band of several pixels to one of the edges of the chips ${ }^{9}$ were excluded from our catalogs.

The saturated stars ${ }^{10}$ were not included in the catalogs because of the uncertainty in their photometry.

\footnotetext{
${ }^{6}$ The following values have been calculated from the image identified by $($ run, rerun, camcol, field $)=(1889,40,3,105)$ which is the one taken in most favorable conditions.

${ }^{7}$ In single-image mode the detection and the photometry are done using the same image. In dual-image mode the detection is done in one reference image and the photometry of the detected objects is done in a second image.

${ }^{8}$ Nowadays, the use of weighted images is widely used and a safe procedure, however, at the moment of constructing the catalogs we saw that the use of the weighted images increased the uncertainties in the magnitudes so we opted for not using them.

9 The bands were constructed interactively to ensure that the problematic regions were avoided.

10 The exposure time of the single exposures were set to avoid the saturation level for galaxies so no galaxy was found to reach such a level.
}

Once having obtained the photometry in both bands, we applied the color term correction of the flux calibration. This was done using the color index $(B-V)$ measured in apertures of $R=5 \mathrm{kpc}$, except for objects with smaller linear radius for which smaller apertures $(R=2 \mathrm{kpc})$ were used.

For each field, SExtractor was run on the images obtained for both the bright and faint galaxies (see Sect. 2.1) and the final catalog was obtained by merging the two catalogs.

\subsection{Star-galaxy classification}

The star-galaxy classification was made relying upon the stellarity index (CLASS_STAR) computed by SExtractor which ranges from 0 (galaxies) to 1 (stars). We decided to make a rather robust selection in two groups, "stars" and "galaxies", with the highest probability of being correct. Objects not fitting those criteria were left in a third group of "unknown" classification. Practically we imposed the following criteria:
Stars
Galaxies
CLASS_STAR $\geq 0.8$
Unknown
CLASS_STAR $\leq 0.2$

In spite of the robustness of the criteria that we used, the final catalogs were checked for possible star/galaxy misclassifications. Since the number of objects was too large for any individual analysis, we opted for using several plots of different combinations of parameters to detect these possible misclassifications. In Fig. 2 we show some of the combinations of parameters that separate stars from galaxies:

- $V-\mu_{\max }$, where $\mu_{\max }$ is the surface brightness of the brightest pixel in an object and $V$ is the total $V$ band apparent magnitude which actually is SExtractor's MAG_AUTO;

- $\log _{10}$ (Isophotal Area) $-V$, where Isophotal Area is the area in pixels of each object above the threshold;

- $\log _{10}$ (Isophotal Area) - $\log$ (FWHM);

- $V-\left(V\left(r \leq 1^{\prime \prime}\right)-V\right)$, where $V\left(r \leq 1^{\prime \prime}\right)$ is the magnitude measured in an aperture of radius $=1^{\prime \prime}$.

In all these diagrams, stars populate a narrow and well defined region while galaxies are more spread throughout the plane. This clear segregation decreases for smaller and fainter objects for which stars and galaxies populate similar regions in the diagrams. Therefore, these diagrams are not really useful for faint $(V \gtrsim 20)$ and small objects $(\log ($ Area(pixels) $) \lesssim 2)$.

Displaying these kinds of diagrams for each cluster field it was easy to detect outliers that most probably represented objects with a wrong classification as well as remaining spurious detections. The (very few) suspicious objects were then visually checked and their classification changed if needed.

At the end of this process we computed the degree of misclassification of the original SExtractor catalogs. It was found that the fraction of misclassification was minimal, less than $1 \%$ of misclassified stars and $<0.6 \%$ of misclassified galaxies, up to $V \sim 22$. Therefore, we consider that the remaining misclassifications in this range of magnitude should be even smaller. For fainter objects the previous diagrams lose their utility since stars and galaxies populate almost the same region and even the visual check becomes useless.

The published catalogs will be regularly updated to correct for possible new spurious objects or misclassifications found so users are encouraged to use the latest version that will be published in WINGS web site: http://web.oapd.inaf.it/ wings/. 

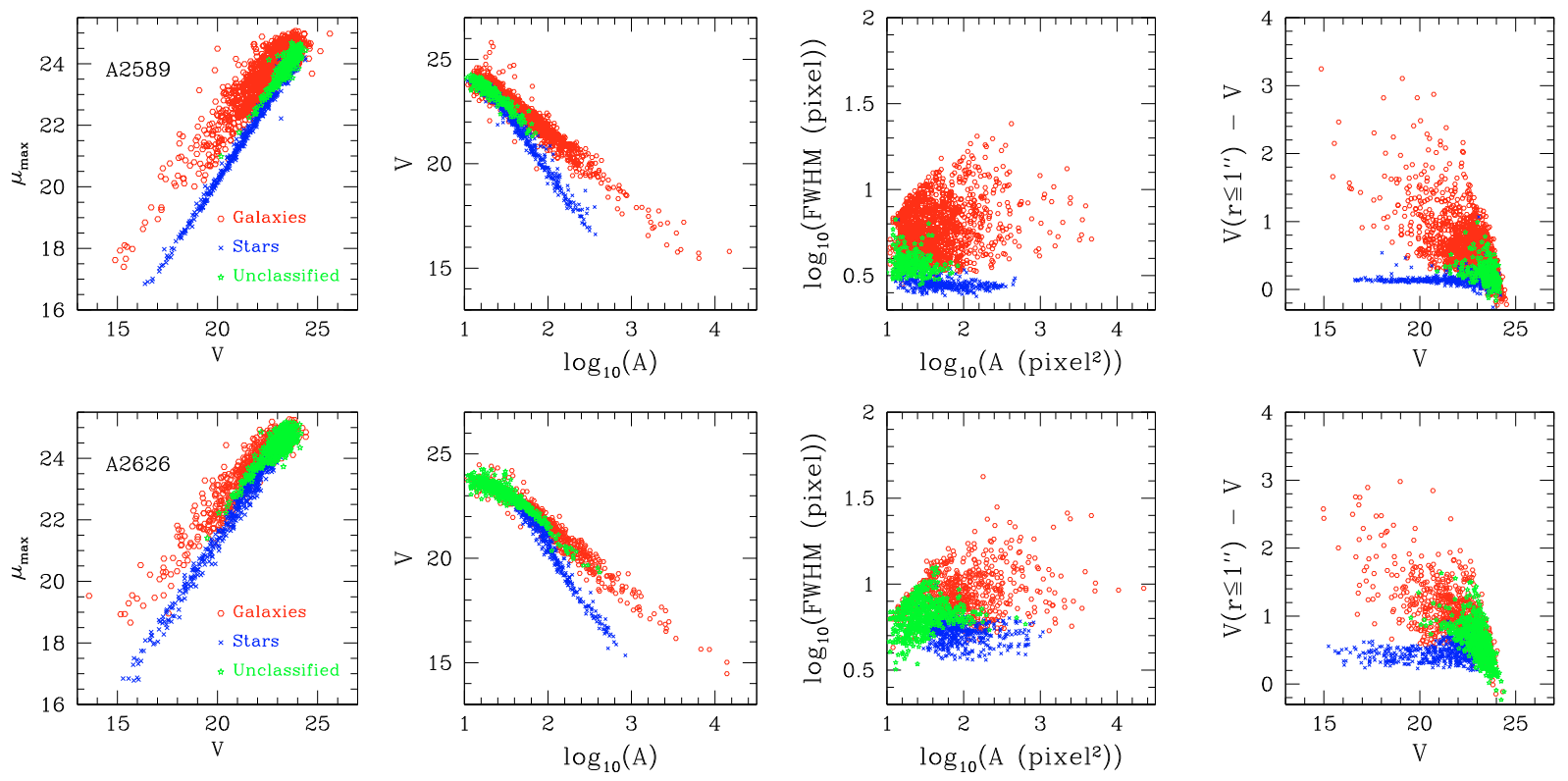

Fig. 2. Four different kinds of diagrams which can be used to perform a star/galaxy separation. From left to right: $\mu_{\max }-V$; V $-\log ($ Area); $\log (\mathrm{FWHM})-\log ($ Area $) ; V-\left(V\left(r \leq 1^{\prime \prime}\right)-V\right)$. The data come from two fields with quite different seeing conditions: A2589 $\left(\mathrm{FWHM}_{*}=0 .{ }^{\prime \prime} 85 ;\right.$ upper panels) and A2626 $\left(\mathrm{FWHM}_{*}=1\right.$.'.64; lower panels). Red squares are galaxies, blue crosses stars and green dots unclassified objects. For better visualization, only $20 \%$ of the objects have been plotted.

\section{The catalogs}

\subsection{Catalogs description}

We have constructed three catalogs for each field containing respectively galaxies, no saturated stars and objects of unknown classification. The structure of all of them is the same and an example is shown in Table 1.

The parameters stored for each object are the following (in parentheses we give the name of the output parameter from SExtractor used to calculate them):

- Equatorial coordinates (J2000.0) of the barycenter (X_IMAGE, Y_IMAGE) and of the peak of emission (XPEAK_IMAGE, YPEAK_IMAGE), taken from the $V$ band image.

- Isophotal area (ISOAREA_IMAGE).

- Kron radius (KRON_RADIUS).

- Full width at half maximum (FWHM_IMAGE).

- Axis ratio computed as the ratio of the SExtractor's parameters B_IMAGE and A_IMAGE.

- Position angle with respect to the North and measured counter-clockwise (THETA_IMAGE).

- SExtractor's FLAG.

- SExtractor's stellarity index (CLASS_STAR).

- Concentration index measured as the ratio between the flux in the central $9 \%{ }^{11}$ of the pixels and the total flux (CONCENTRATION).

- Contrast index measured as the ratio between the flux in the brightest $30 \%$ of the pixels and the total flux (CONTRAST).

- Surface brightness of the brightest pixel (MU_MAX).

- And the following magnitudes in both bands:

- Total magnitudes: SExtractor's MAG_ISO, MAG_ ISOCOR and MAG_AUTO.

11 Due to a bug found in the modified version of SExtractor used to compute the concentration index, the flux is not measured in the central $30 \%$ of the pixels but in the central $9 \%$.
- Three magnitudes at fixed physical apertures (at the target cluster's redshift): $R=2 \mathrm{kpc}, 5 \mathrm{kpc}, 10 \mathrm{kpc}$.

- Three magnitudes at fixed angular apertures: 1.'6, 2'.0, 2.. $16^{12}$.

The photometric catalogs are public only in electronic format via CDS and at WINGS' website http://web.oapd.inaf. it/wings/. The reduced images in both bands will be made available upon request to the authors.

In the following we give indications about some points to be taken into account when using the catalogs.

Coordinates: the astrometry of the images is discussed in Appendix A.4 of Paper $\mathrm{I}^{13}$. The overall quality is very satisfactory (see Appendix A.4 of Paper I) with uncertain-

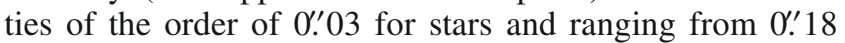
for WFI@ESO images to 0.'25 for WFC@INT ones. For each object two positions have been computed: that of the barycenter of the light distribution and that of the peak of the emission. For most objects, they are almost coincident. However there are two situations in which it is better to use one and not the other. First, for faint low surface brightness galaxies the barycenter position is preferable since the position of the peak is highly affected by the noise. On the other hand and most importantly, for large extended galaxies (such as the brightest cluster galaxies) the coordinates of the peak emission are a better choice to locate the center of the object because the barycenter is strongly affected by the shape of the most external isophotes.

Shape parameters: care must be taken when using the shape parameters especially for objects in crowded fields and/or

\footnotetext{
12 These fixed apertures have been chosen for the multifiber spectroscopy of the WINGS fields. 1." 6 is the projected diameter of the fibers in Autofib2@WHTwhile in the 2dF@AAT the diameter varies radially in the field from 2 '. 16 in the center to $2{ }^{\prime \prime} 0$ in the edges.

13 The astrometry was done with respect to the USNO-A2 Catalogs which result in slight differences $(\lessgtr 0$ " 3 ) with respect the USNO-B1.
} 
Table 1. Example of entries in one of the photometric catalogs.

\begin{tabular}{ccccccccccccc}
\hline \hline ID & $\begin{array}{c}\alpha_{\text {Bary }} \\
(\mathrm{deg})\end{array}$ & $\begin{array}{c}\delta_{\text {Bary }} \\
(\mathrm{deg})\end{array}$ & $\begin{array}{c}\alpha_{\text {Peak }} \\
(\mathrm{deg})\end{array}$ & $\begin{array}{c}\delta_{\text {Peak }} \\
(\mathrm{deg})\end{array}$ & $\begin{array}{c}\text { Area } \\
\left(\operatorname{arcsec}^{2}\right)\end{array}$ & $\begin{array}{c}r_{\text {Kron }} F W H M \\
\left({ }^{\prime \prime}\right)\end{array}$ & $\begin{array}{c}\left({ }^{\prime \prime}\right) \\
\text { W }\end{array}$ & $\begin{array}{c}\text { PA } \\
(\mathrm{deg})\end{array}$ & FLAG S.I. & \\
\hline WINGS J004135.4-090100.5 & 10.39751 & -9.01681 & 10.39751 & -9.01682 & 17.39 & 1.49 & 6.26 & 0.73 & 63 & 19 & 0.02 & $\ldots$ \\
WINGS J004106.0-090104.2 & 10.27495 & -9.01783 & 10.27500 & -9.01781 & 41.86 & 0.83 & 2.03 & 0.75 & 31 & 0 & 0.03 & $\ldots$ \\
\hline
\end{tabular}

\begin{tabular}{|c|c|c|c|c|c|c|c|c|c|c|c|c|}
\hline \multicolumn{13}{|c|}{$\bar{l} V$ band } \\
\hline Conc. & Contr. & $\mu_{\max }$ & MAG_ISO & MAG_ISOCOR & MAG_AUTO & $\operatorname{MAG}(2 \mathrm{kpc})$ & MAG $(5 \mathrm{kpc})$ & $\operatorname{MAG}(10 \mathrm{kpc})$ & $\operatorname{MAG}\left(1^{\prime \prime} 6\right)$ & $\operatorname{MAG}\left(22^{\prime \prime}(0)\right.$ & $\operatorname{MAG}(2 . " 16)$ & \\
\hline $\begin{array}{ll}\ldots & 0.384\end{array}$ & 0.543 & 22.97 & 20.71 & 20.44 & 19.86 & 21.43 & 20.63 & 20.10 & 22.53 & 22.19 & 22.09 & \\
\hline .. 0.460 & 0.777 & 21.03 & 18.58 & 18.55 & 18.57 & 19.11 & 18.60 & 18.51 & 20.48 & 20.09 & 19.97 & \\
\hline
\end{tabular}

\begin{tabular}{cccccccccc}
\hline \hline \multicolumn{10}{c}{$B$ band } \\
\multicolumn{10}{c}{ MAG_ISO } \\
\hline$\ldots$ & 21.41 & 20.46 & 20.00 & 22.18 & 21.09 & 20.21 & 23.37 & 23.01 & 22.90 \\
$\ldots$ & 19.60 & 19.48 & 19.58 & 20.16 & 19.61 & 19.47 & 21.58 & 21.18 & 21.05 \\
\hline
\end{tabular}

ID: object internal identification.

$\left(\alpha_{\text {Bary }}, \delta_{\text {Bary }}\right)$ : equatorial coordinates (J2000.0) of the barycenter.

$\left(\alpha_{\text {Bary }}, \delta_{\text {Bary }}\right)$ : equatorial coordinates (J2000.0) of the brightest pixel.

Area: area above the detection threshold.

$r_{\text {Kron }}$ : Kron radius used to computed the MAG_AUTO magnitude.

FWHM: full width at half maximum assuming a Gaussian core.

$b / a$ : axis ratio.

PA: position angle of the major axis (North $=0^{\circ}$, counter-clockwise).

FLAG: SExtractor's FLAG.

S.I.: SExtractor's stellarity index CLASS_STAR.

$\mu_{\max }$ : surface brightness of the brightest pixel.

Conc.: Abraham's concentration index, measured as the ratio between the flux in the central $9 \%$ of the pixels and the total flux.

Contr: Abraham's contrast index, measured as the ratio between the flux in the brightest $30 \%$ of the pixels and the total flux.

MAG_ISO: SExtractor's isophotal magnitude.

MAG_ISOCOR: SExtractor's isophotal corrected magnitude.

MAG_AUTO: SExtractor's Kron aperture magnitude.

$\operatorname{MAG}(2 \mathrm{kpc})$, MAG $(5 \mathrm{kpc}), \operatorname{MAG}(10 \mathrm{kpc})$ : magnitudes within apertures of radius $2 \mathrm{kpc}, 5 \mathrm{kpc}$ and $10 \mathrm{kpc}$, respectively, measured at the clusters' redshift.

$\operatorname{MAG}\left(11^{\prime \prime} 6\right), \operatorname{MAG}\left(22^{\prime \prime}()\right), \operatorname{MAG}\left(22^{\prime \prime} 16\right)$ : magnitudes measured within apertures of diameter equal to $1^{\prime \prime} 6,22^{\prime \prime} 0,2{ }^{\prime \prime} 16$, respectively.

contaminated by close companions. The use of the FLAGS values can help to check this.

Total magnitudes: SExtractor gives four different measures of total magnitude. MAG_ISO is the integrated light above the detection threshold. MAG_ISOCOR is an attempt to correct for the light lost in the wings of the objects making use of a Gaussian model approximation. It was found that for $B$ band magnitudes computed in dual mode MAG_ISOCOR can give wrong values so we discourage its usage. MAG_AUTO is another attempt to guess the total magnitude of the galaxies using Kron apertures (Kron 1980; Infante 1987; Bertin \& Arnouts 1996). However, several works (e.g. Franceschini et al. 1998) have shown the problems of this approximation, especially its dependence on the light profile of the object. In Sect. 3.2.1 we will show that while for stellar and exponential profiles the MAG_AUTO magnitude is a good approximation, for de Vaucouleurs profiles there is a systematic offset of $\sim 0.2^{\mathrm{m} 14}$. Finally, SExtractor includes another total magnitude called MAG_BEST magnitude. This corresponds to MAG_AUTO except in those cases in which the light of the object has a contamination by external sources higher than $10 \%$, when MAG_ISOCOR is used instead. Several sources including the last SExtractor's Users Guide discourage

\footnotetext{
14 A detailed explanation of the Kron magnitude and its problems can be found in Graham \& Driver (2005)
}

the usage of MAG_BEST in favor of MAG_AUTO. Our experience when constructing the catalogs has convinced us that MAG_AUTO gives the best approximation to the total magnitude of objects.

Saturated stars: since the aim of the catalogs is the study of clusters of galaxies, the saturated stars were discarded. This should be taken into account when using our catalog of stars. In the case of WFC@INT fields the incompleteness produced by saturation affects stars brighter than $V \sim 17$, while for WFI@ESO fields this limits is $V \sim 16$, the difference mainly from the different pixel size.

In addition to the information on the single objects found in each field, in Appendix D the reader can find a set of tables with additional information about the target clusters, peculiarities of the fields and the conditions of observation that could be useful when working with our published data.

\subsection{Quality checks}

In this section we describe the internal photometric quality ${ }^{15}$ of the data and the accuracy of the star/galaxy classification in our catalogs.

\footnotetext{
15 The comparison with external data (SDSS) was already done in Paper I, Sect. 5.4.
} 


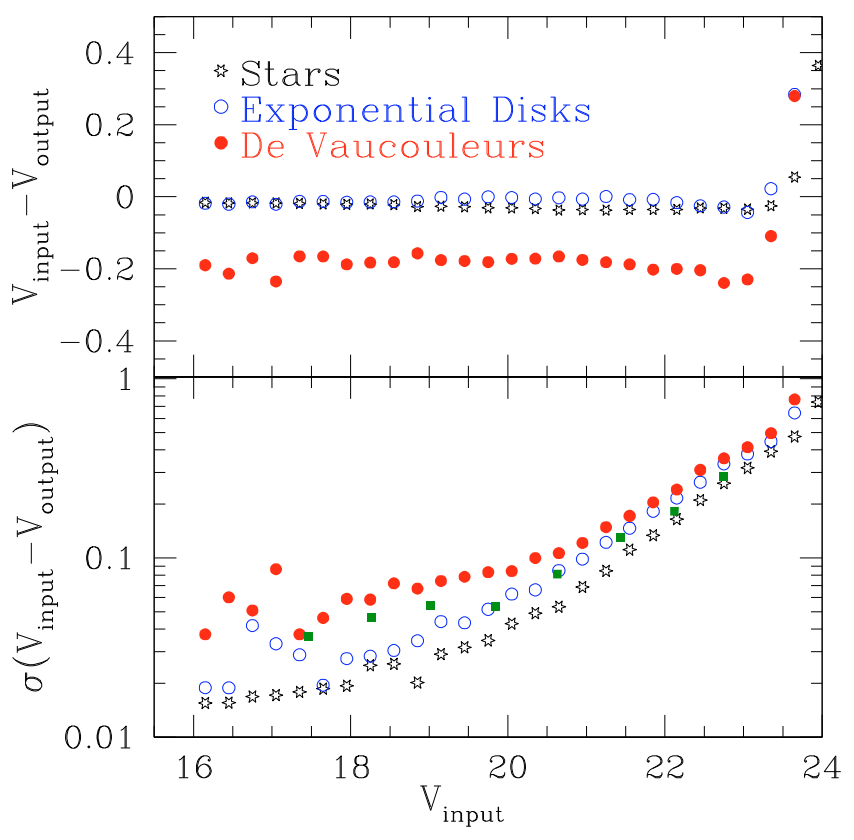

Fig. 3. Photometric errors computed from simulations. Upper panel: systematic offset of the measured magnitude ( $\left.V_{\text {output }}\right)$ with respect to the input magnitude $\left(V_{\text {input }}\right)$ as a function of the input magnitude. Lower panel: dispersion of this difference. Green squares show the differences between the photometry of two fields (A780 and A970) observed with WFC@INT and WFI@ESO (same as lower fifth panel of Fig. 11 in Fasano et al. (2006).

\subsubsection{Photometric errors}

When running without weighted images, SExtractor computes just the photon-noise errors of the magnitudes. For our deep images these values are unrealistically small and for this reason we do not report such errors in our catalogs. In fact, the errors in the photometry are mostly related to local background variations or to contamination by the light of close companions. To estimate these uncertainties we performed simulations in which synthetic stars as well as galaxies with exponential and de Vaucouleurs profiles were inserted in the original images. Figure 3 (upper panel) shows the differences found between the input magnitude ( $\left.V_{\text {input }}\right)$ and that measured by SExtractor ( $\left.V_{\text {output }}\right)$ as a function of the input magnitude for the three types of objects used in the simulations: stars and the two types of galaxy models. Each point is the central biweight estimator (Beers et al. 1990) of the difference $\left(V_{\text {input }}-V_{\text {output }}\right)$ for objects in an interval of $\Delta\left(V_{\text {input }}\right)=0.3^{\mathrm{m}}$. In the lower panel we show the scale biweight estimator of $V_{\text {input }}-V_{\text {output }}$ in the same intervals. Note the use of a logarithmic scale in the ordinate axis.

It is clear that for de Vaucouleurs profiles, SExtractor produces total magnitudes that are $\sim 0.2^{\mathrm{m}}$ fainter than real ones, or equivalently, fluxes that are $\sim 20 \%$ lower. This is a known problem of SExtractor (Franceschini et al. 1998; Benítez et al. 2004) although the differences reported between the real and the measured magnitudes vary from work to work. Contrary to previous works, we find that the difference between input and output magnitudes remains almost constant up to our detection limit $(V \sim 23)$. On the other hand, stellar and exponential profiles show good agreement with mean differences $\$ 0.05^{\mathrm{m}}$.

The simulations also give us a more realistic measure of the uncertainties on the magnitudes (lower panel of Fig. 3). At fixed input magnitude, the largest uncertainties are for galaxies with de Vaucouleurs profiles and the lowest are for stars. The trend

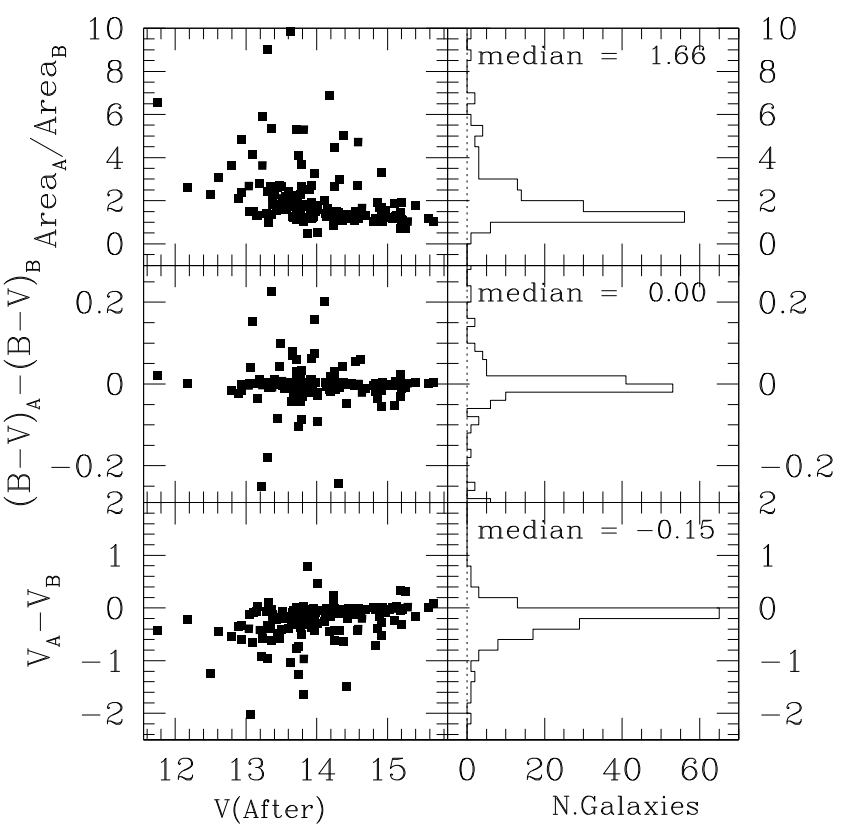

Fig. 4. Comparison of the values of the total magnitude (lower panels), color index $(B-V)$ (middle panels) and isophotal area (upper panels) measured by SExtractor of the modeled galaxies before and after separating them from the rest of the objects. Left: distribution of the differences of these parameters as a function of the total $V$ magnitude. Right: distribution of the same differences. Index " $\mathrm{B}$ " denotes the values before applying our procedure and index "A" indicates the values after applying our procedure.

with input magnitude is similar for the three types of objects with a steeper increase of the uncertainties at magnitudes fainter than $V \sim 20$. As a comparison the figure also shows (green points) the differences in magnitudes found when comparing the data from two fields (A780 and A970) observed with our two instrumental set-ups (see Fig. 11 from Fasano et al. 2006). Therefore, the typical uncertainties in our catalogs up to $V \sim 21.5$ is less than $0.1 \mathrm{mag}$. For magnitudes fainter than $V=21.5$, it is possible to estimate the typical uncertainties with the following expressions:

$$
\begin{aligned}
& \sigma_{V}(\text { Star })=10^{0.309 V-7.62} \\
& \sigma_{V}(\text { Exp })=10^{0.271 V-6.67} \\
& \sigma_{V}(\text { deVauc })=10^{0.259 V-6.34}
\end{aligned}
$$

\subsubsection{Photometry of the modeled objects}

In this section we check the improvement in the photometry of the objects modeled after applying the procedure described in Appendix A to the images.

Figure 4 shows the effect of the model subtraction on the output from SExtractor of some photometric parameters of the modeled galaxies. The large increase in the isophotal area after applying our procedure clearly reflects the issue with the segmentation of large galaxies (see Fig. 1b). This also affects the computation of the total magnitudes, as can be seen in the lower panel of Fig. 4. On the other hand, the color index $(B-V)$ shows smaller variations because of the use of aperture magnitudes to compute it.

In addition to the improvement in the photometry of the subtracted objects we also increased the detection rate of objects projected onto the modeled halos. In the case of objects projected onto the halos of the BCGs we found $\sim 16 \%$ more 


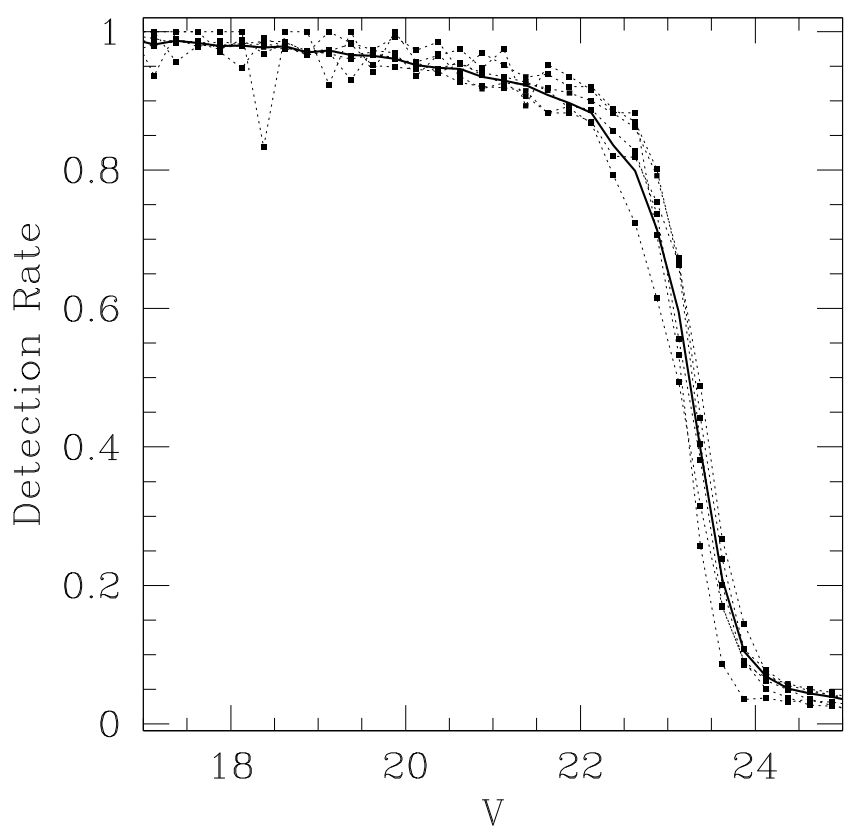

Fig. 5. Average detection rate in each observing run computed from simulations. The stronger black line is the detection rate averaged over the 77 fields.

objects after the subtraction of the BCG. This is a relevant result especially in studies of the spatial distribution of galaxies in the cluster.

\subsubsection{Completeness and goodness of the star/galaxy classification}

The simulations described above were originally intended also to check the detection rate (completeness) and the success rate of the star/galaxy classification. However, we realized that the second step could not be done due to an unexpected issue. We found that commonly used programs to simulated objects (stars and galaxies) do not work well at faint magnitudes. The result is that when running SExtractor, simulated stars have a lower probability of being well classified than real stars (see Appendix B). For that reason, simulations were used only to estimate the detection rate in each field.

Figure 5 shows the average detection rate run by run (dotted curves) and the total average detection rate (continuous line). The curves are quite similar and we can considered our global catalogs $90 \%$ complete for objects with $V \lesssim 21.7$. The $50 \%$ detection level is reached at $V \sim 23.2$. In addition, we provide in Table D.2 the $V$ band magnitudes at which the detection rate drops to $90 \%, 75 \%$ and $50 \%$ in each field as well as the surface brightness detection thresholds in the same band.

As an external check of the completeness computed with the simulations we took the image of the center of A1795 taken from the Space Telescope and compared the detection rates in that image with ours. The results are presented in Appendix $C$ and they are in good agreement with those from the simulations.

Concerning the star/galaxy classification, for objects with $V \lesssim 21$ the difference between light profiles of stars and galaxies is large enough to allow SExtractor to distinguish them easily and, moreover, the diagrams like those in Fig. 2 can be used to reclassify the cases where SExtractor fails. Therefore, for these bright objects the number of misclassifications can be considered negligible.

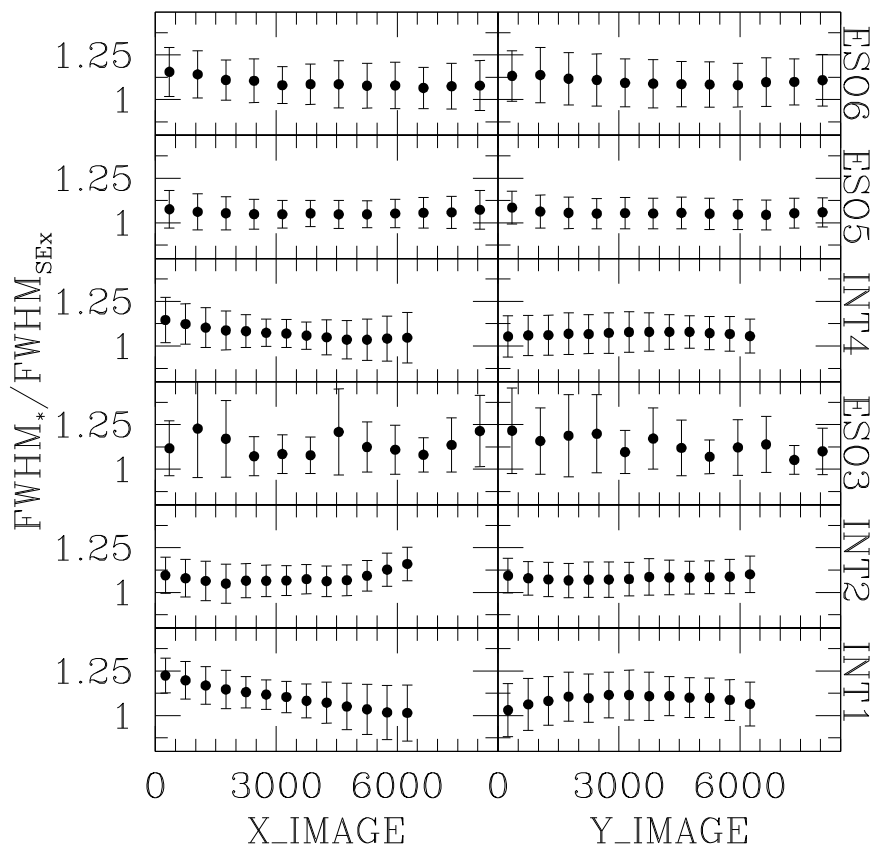

Fig. 6. Spatial variation of the stellar FWHM in the different runs of WINGS. Each point is the central biweight estimator (CBE, Beers et al. $1990)$ of the ratio between the $F W H M\left(F W H M_{*}\right)$ and the $F W H M$ used as input in SExtractor $\left(F W H M_{\mathrm{SEx}}\right)$ for stars in bins of 500 pixels (WFC@INT) or 700 pixels (WFI@ESO) along both axis. The errors have been computed as the scale biweight estimator (SBE).

The problem arises for fainter objects whose profiles become more similar and small variations in the local conditions (e.g. small variations in the background) can make SExtractor fail to perform a good classification. Moreover, the number of objects is so large that interactive cleaning is unpractical and even the visual check cannot help. This is worsened by the fact that the point spread function actually varies throughout the field (Fig. 6). For these reasons, a statistical approach appears to be the best option to check the reliability of the star/galaxy classification at faint magnitudes. As stated above, the simulations were found to be unreliable at faint magnitudes so we opted for another way to estimate the goodness of our star/galaxy classification as well as to guess the composition of the "unknown" class of objects. First of all, we can compare the fraction of objects in each group (stars/galaxies/unknown) as a function of the $V$ magnitude (see Fig. 7). As expected, the fraction of unknown objects increases at fainter magnitudes. This increase rises more steeply for $V \gtrsim 22$, i.e. when the completeness starts to fall rapidly. A interesting point is that this rise corresponds to a change in the trend of the fraction of galaxies, pointing to the fact that most unknown objects should be galaxies. Another way to see this is by relying upon external sources for galaxy and star counts. For the galaxies we took data from the ESO-Spitzer Imaging extragalactic Survey (ESIS, Berta et al. 2006) while for the stars we used the models of distribution of stars in the Galaxy of the Observatory of Besançon ${ }^{16}$ (Robin et al. 2003). Of course, this check is relevant only in the faintest part of the magnitude distribution where counts from stars and background galaxies dominate those of the target cluster. With both counts we computed the fraction of galaxies with respect to the total number of objects as a function of the apparent magnitude (continuous line in Fig. 8). This is compared with the same fraction measured from

16 http://bison.obs-besancon.fr/modele/ 


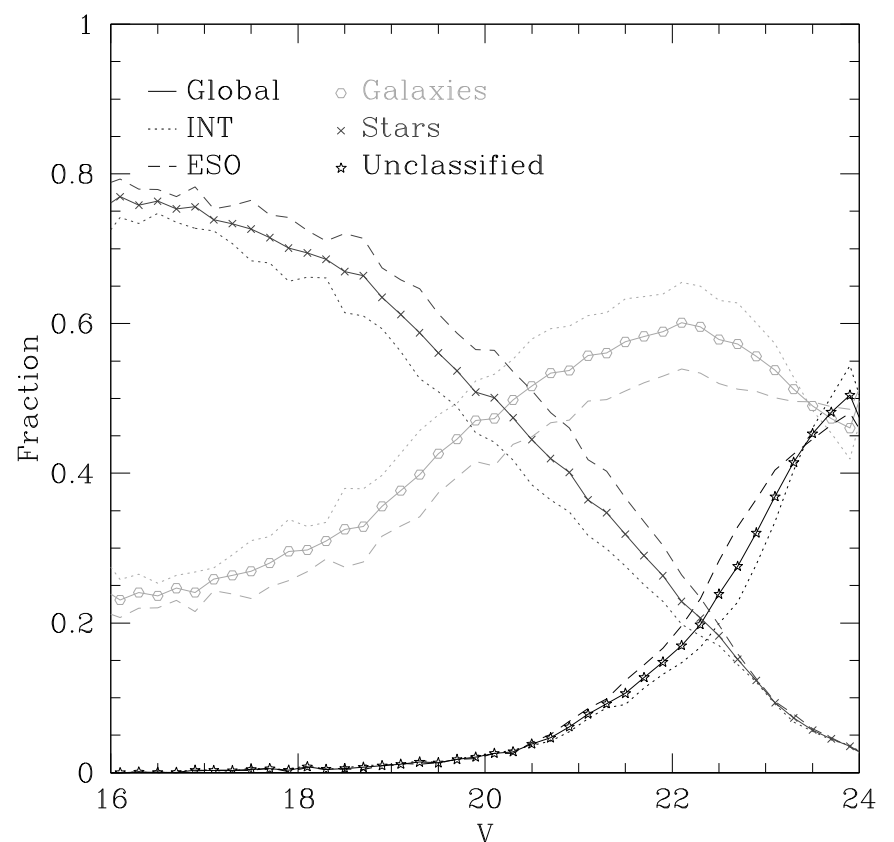

Fig. 7. Relative numbers of the three types of objects: galaxies, stars and unknown objects. WFI@ESO fields show a higher fraction of stars because some of them point closer to the direction of the galactic center.

WINGS fields (dashed lines) although measured in off-center regions to avoid the high contribution of galaxies from the center of the clusters. If the "unknown" objects are ignored (shortdashed line in the figure) the fraction of galaxies observed in our fields becomes smaller than that observed in the ESIS+Besançon sample for $V \gtrsim 20.5$. This is an unexpected result since our fields include the contribution of the cluster. To check the influence of the classification of galaxies as "unknown" objects, we assumed that all the "unknown" objects were galaxies and recomputed the fraction (long-dash line). This produces an overestimation of the fraction of faint galaxies. It is possible to compute the minimum fraction of galaxies in the "unknown" sample which makes the WINGS galaxy fraction equal that of the background counts of galaxies and stars. This is shown in the upper panel of Fig. 8 and for $V>23$ this fraction is greater than $80 \%$.

The agreement between the fraction of galaxies observed and expected from external sources reassures us that even at very faint magnitudes $(V \sim 24)$ the star/galaxy classification is not a mere random assignment from which one would expected half stars and half galaxies. Since the difference in the fraction of galaxies is always $<10 \%$ between our data and those from the external sources we can assume that statistically the star/galaxy classification holds quite well up to $V \sim 24$.

\section{Summary}

In this paper we present the first data release from the WINGS project. We have produced photometric catalogs of all the objects found in 77 fields centered on nearby clusters. The objects have been classified as galaxies, stars and objects of unknown type. For each object we give positions, geometrical parameters and different kinds of total and aperture magnitudes in both observed bands, $B$ and $V$.

Our catalogs are $90 \%$ complete at $V \sim 21.7$ and $50 \%$ at $V \sim 23.2$ although these values vary from field to field, especially depending on the total exposure time. Our star/galaxy classification relies on the CLASS_STAR parameter of SExtractor

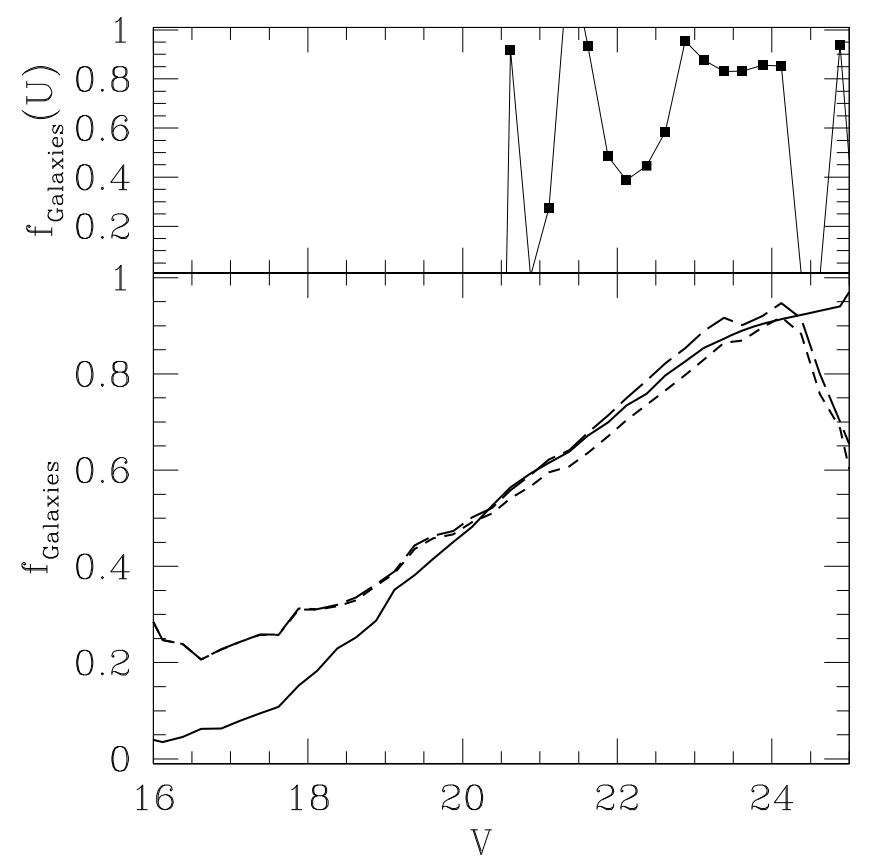

Fig. 8. Lower panel: average fraction of galaxies as a function of the $V$ magnitude. Continuous line: fraction computed assuming the stellar counts from the models of Besançon and galaxy counts from Berta et al. (2006). Short dashed line: data from off-center regions ignoring the unknown objects. Long dashed line: data from off-center regions including the unknown objects as galaxies. Upper panel: minimum fraction of galaxies in the unknown objects.

but we have performed a visual checking of the brightest objects $(V \lesssim 22)$ that makes the misclassifications negligible. For the faintest objects we tried to use simulations but we found that these were not reliable in the appropiate range of brightness $(V>$ 22). However, when we compare the fraction of galaxies found in our catalogs with those from external sources (ESIS' deep galaxy counts and stars counts from Observatory of Besançon's Galaxy Model), we find that both are compatible within $\pm 10 \%$. This result makes us confident in our star/galaxy classification even at faint magnitudes, at least statistically. Again, the reliability of the classification varies from field to field, depending on the seeing and also on the spatial variation of the PSF.

A notable feature of these catalogs is that extended galaxies have been treated in a special manner which has allowed us to improve their photometry, with several cases in which the total magnitudes were initially underestimated by more than one magnitude. This is a critical issue for our fields, since our clusters are often centered on very bright, extended galaxies, which are themselves important targets of our study. We have been able not only to improve the photometry of these galaxies but also to detect $\sim 16 \%$ more objects around the BCG relative to the case in which the galaxy is not subtracted.

In addition to the catalogs we also release the reduced images in both bands $(B, V)$ and the preprocessed images with the large halos removed as well as the images with only the largest galaxies.

The scientific analysis of these data will be published in the subsequent papers of this series.

Acknowledgements. The authors acknowledge the referee for the useful comments that helped to improve the initial manuscript. J. Varela acknowledges a post-doc fellowship FIRB/MIUR from the Ministero dell'Istruzione, dell'Università e della Ricerca (Italy) and a "Juan de la Cierva" post-doc fellowship granted by the Spanish "Ministerio de Educación y Ciencia". 
M.M. acknowledges support form the Spanish Ministerio de Educación y Ciencia, grants AYA2002-01241, AYA2005-07789 and AYA2006-14056. The $2.5 \mathrm{~m}$ "Isaac Newton" Telescope located at Roque de los Muchachos (La Palma, Spain) is operated by the Isaac Newton Group of Telescopes on behalf of the Particle Physics and Astronomy Research Council (PPARC) of the United Kingdom. The observations at the INT telescope were done under "International Time Allocation". The $2.2 \mathrm{~m}$ Telescope located at La Silla (Chile) is on loan to European Southern Observatory from the Max Planck Gesellshaft (Germany). This research has made use of the NASA/IPAC Extragalactic Database (NED) which is operated by the Jet Propulsion Laboratory, California Institute of Technology, under contract with the National Aeronautics and Space Administration. IRAF (Image Reduction and Analysis Facility) is written and supported by the IRAF programming group at the National Optical Astronomy Observatories (NOAO) in Tucson, Arizona. NOAO is operated by the Association of Universities for Research in Astronomy (AURA), Inc. under cooperative agreement with the National Science Foundation.

\section{References}

Abell, G. O. 1957, The distribution of rich clusters of galaxies, A catalogue of 2712 rich clusters found on the National Geographic Society Palomar Observatory Sky Survey (Chicago: University of Chicago Press)

Abell, G. O. 1959, Leaflet of the ASP, 8, 121

Abraham, R. G., Valdes, F., Yee, H. K. C., \& van den Bergh, S. 1994, ApJ, 432, 75

Abraham, R. G., van den Bergh, S., Glazebrook, K., et al. 1996, ApJS, 107, 1

Bahcall, N. A., McKay, T. A., Annis, J., et al. 2003, ApJS, 148, 243

Beers, T. C., Flynn, K., \& Gebhardt, K. 1990, AJ, 100, 32

Benítez, N., Ford, H., Bouwens, R., et al. 2004, ApJS, 150, 1

Berta, S., Rubele, S., Franceschini, A., et al. 2006, A\&A, 451, 881

Bertin, E., \& Arnouts, S. 1996, A\&AS, 117, 393

Biviano, A., Katgert, P., Mazure, A., et al. 1997, A\&A, 321, 84
Butcher, H., \& Oemler, A. 1978, ApJ, 219, 18

Cava, A., et al. 2008 [arXiv: 0812 .2022]

De Propris, R., Couch, W. J., Colless, M., et al. 2002, MNRAS, 329, 87

Dressler, A. 1980, ApJS, 42, 565

Dressler, A., Oemler, A., Jr., Couch, W. J., et al. 1997, ApJ, 490, 577

Ebeling, H., Voges, W., Bohringer, H., et al. 1996, MNRAS, 281, 799

Ebeling, H., Edge, A. C., Bohringer, H., et al. 1998, MNRAS, 301, 881

Ebeling, H., Edge, A. C., Allen, S. W., et al. 2000, MNRAS, 318, 333

Fasano, G., Poggianti, B. M., Couch, W. J., et al. 2000, ApJ, 542, 673

Fasano, G., Marmo, C., Varela, J., et al. 2006, A\&A, 445, 805 (Paper I) Franceschini, A., Silva, L., Fasano, G., et al. 1998, ApJ, 506, 600

Goto, T., Sekiguchi, M., Nichol, R. C., et al. 2002, AJ, 123, 1807

Graham, A. W., \& Driver, S. P. 2005, PASA, 22, 118

Gunn, J. E., \& Gott, J. R. I. 1972, ApJ, 176, 1

Infante, L. 1987, A\&A, 183, 177

Katgert, P., Mazure, A., Perea, J., et al. 1996, A\&A, 310, 8

Kron, R. G. 1980, ApJS, 43, 305

Kurk, J. D., Pentericci, L., Overzier, R. A., et al. 2004, A\&A, 428, 817

Lubin, L. M., Oke, J. B., \& Postman, M. 2002, AJ, 124, 1905

Miller, C. J., Nichol, R. C., Reichart, D., et al. 2005, AJ, 130, 968

Oemler, A. J. 1974, ApJ, 194, 1

O’Hely, E., Couch, W. J., Smail, I., Edge, A. C., \& Zabludoff, A. 1998, PASA, 15,273

Pimbblet, K. A., Smail, I., Edge, A. C., et al. 2001, MNRAS, 327, 588

Postman, M., Franx, M., Cross, N. J. G., et al. 2005, ApJ, 623, 721

Robin, A. C., Reylé, C., Derrière, S., \& Picaud, S. 2003, A\&A, 409, 523

Smith, R., Hudson, M., Nelan, J., et al. 2004, AJ, 128, 1558

Steidel, C. C., Adelberger, K. L., Shapley, A. E., et al. 2000, ApJ, 532, 170

Valentinuzzi, T., et al. 2008, in preparation

van Dokkum, P. G., Franx, M., Fabricant, D., et al. 2000, ApJ, 541, 95

Zwicky, F., Herzog, E., \& Wild, P. 1963, Catalogue of galaxies and of clusters of galaxies, Pasadena, California Institute of Technology (CIT) 


\section{Appendix A: Full description of the extended halo removal}

In this Appendix we give a detailed description of the whole procedure followed to remove the objects with extended halos.

This is the outline of the whole process, while the single steps are explained in some detail thereafter:

1. A background map is computed using the original images.

2. The extended objects (galaxies and stars) to be removed are selected interactively.

3. For each selected object, the following steps are performed:

(a) The objects projected onto it are masked ${ }^{17}$ with an interactive procedure.

(b) Its isophotes are fitted with the IRAF task ellipse.

(c) The resulting elliptical isophotes are then used as the input of the task bmodel to construct a model of the extended object.

(d) The model is subtracted from the original image.

(e) In the case of galaxies, when the subtraction leaves residuals due to the structure of the galaxy, these are removed manually with imedit.

4. After removing all the selected objects from the initial image a new background map is calculated.

5. A mask of the remaining objects is constructed with SExtractor. This mask is then used in a second iteration to improve the interactively-made masks (point (3a)) applied during the isophote fitting procedure.

6. At this point, the procedure can be repeated from point (3) until the subtraction is satisfactory.

The process is iterative and could be repeated as many times as necessary but we found that one iteration was enough to reach a good photometric quality.

Now we explain in more detail all the steps of the procedure:

First background computation: due to the variations found from chip to chip and to avoid the influence of the interchip regions (which SExtractor treats as if they were part of the actual image) the images were split into their original chips. After that, an initial background estimation was computed for each chip using SExtractor with BACK_SIZE=256 and BACK_FILTERSIZE $=3$ as input parameter values. To reduce the influence of bright pixels, those with intensities above an established threshold were replaced by the mode of the pixel intensity of the same chip, which can be considered as a first estimation of the background level. The threshold was set manually before starting the process seeking to not remove the bright part of the background.

Once the background was obtained for each chip, the single background images were mosaiced to construct a global background map. This background map was then subtracted from the original image.

Selection of the objects to be removed: the selection of the objects to be modeled and removed was done in a subjective way but following several guidelines. First, the BCG was always modeled. Elliptical galaxies ${ }^{18}$ highly blended and/or

17 The masking procedure consists of creating an auxiliary image or mask that indicates to the fitting program which pixels not include in the fitting procedure. This can be done, for example, by setting to zero all the pixels of the mask except those whose position correspond to pixels in the original image that should not be used in the fit.

${ }_{18}$ Since the process is based on the construction of models of elliptical isophotes it is not suitable for spiral or irregular galaxies.

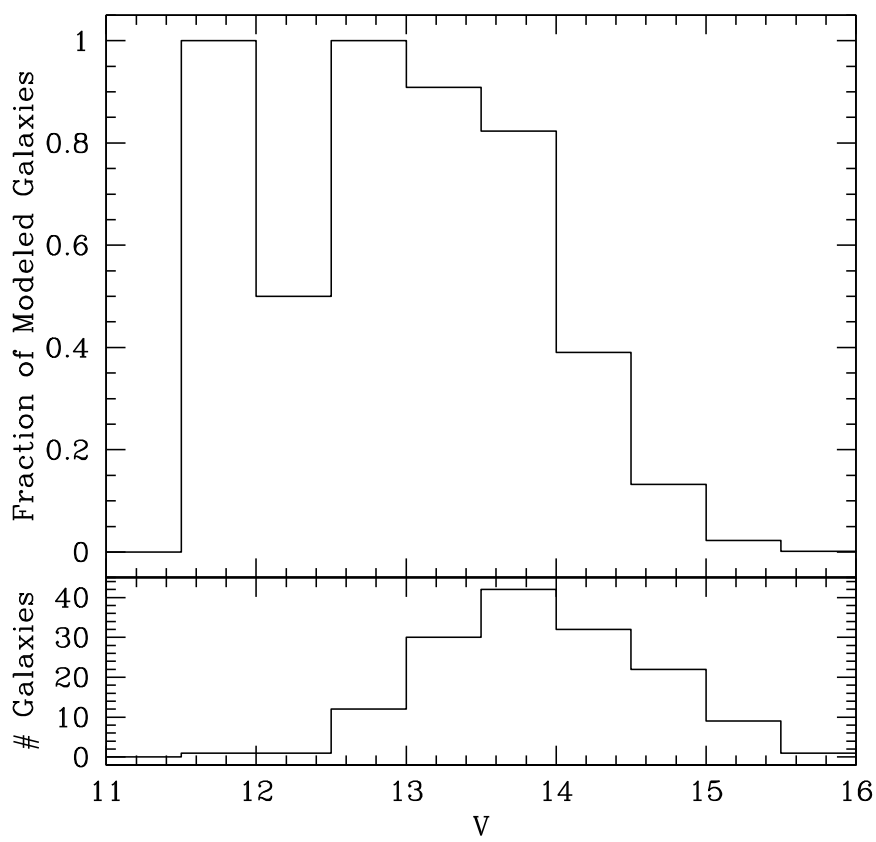

Fig. A.1. Upper panel: fraction of galaxies that were modeled. Lower panel: total number of galaxies that were modeled.

surrounded by small objects were also modeled. The selection of the stars to be modeled was more field-dependent. As the amount of work needed increased substantially with the number of objects to be modeled, in those fields with a high density of stars only the brightest stars were modeled. Sometimes, a star was modeled to avoid the contamination from a close bright galaxy.

Figure A.1 shows the distribution in $V$ of the galaxies that have been processed in this way. Up to $V \sim 14$ most of the galaxies have been modeled.

Fitting and modeling: to reduce the computation time, the process of isophote fitting, modeling and model subtraction was done on small images of the selected objects extracted from the background-subtracted global image.

The isophote fitting was done using the IRAF task ellipse. However, before that, all the projected objects and problematic regions (e.g. interchip regions) were masked. Due to the large number of objects that should be masked and taking advantage of the iterative process, this first step was enough to make a rough mask. Below we will show how the improvement is achieved.

A few differences were introduced when dealing with stars or galaxies. For stars the ellipticity was fixed to zero and the fitting did not reach the innermost region (usually saturated and, therefore, not suitable for fitting isophotes). Also, the center of the isophotes was not fixed because quite often the reflections of the stars were off-center with respect to the central regions. For galaxies no restrictions were imposed.

The output of ellipse was used as the input of the task bmodel which allows us to construct a two dimensional model of the object. This model was subtracted from the original image (i.e. before the first background subtraction). This step was done for all selected objects.

Second background estimation and object mask: the outcome of the previous step was an image similar to the original one, in which large objects have been removed. This allowed us to obtain an improved background map, 
a better background subtracted image and a better detection of the small objects. In fact, using SExtractor with the background-subtracted images after removing the models, it was possible to get a careful mask of all objects (except, of course, from those that were removed from the image). This new mask would serve to refine the previous (manually-done) one.

Second fit and modeling: the fitting process done in the first iteration was repeated using a refined mask for each object. This helped to get a better fit and, as a result, a better model. The main difference with respect to the first iteration is that after the subtraction of the model from the galaxies, sometimes some residuals remained in the central regions. In such cases, these were manually edited using the IRAF task imedit. When editing the images only clearly spurious residuals were replaced by pixels simulating the background signal adding a Gaussian noise whose sigma was computed from surrounding regions. This has no effect on an object's photometry since the photometry of large galaxies is done with another image, avoiding the spurious detection of the residuals.

Construction of the final images: in most fields, only two iterations were enough to achieve a satisfactory result. After modeling all the selected extended objects and subtracting them from the initial image, the background was recomputed again and finally subtracted from the model-subtracted image. The output of this procedure was a backgroundsubtracted image without the extended modeled objects.

The final step was to produce the complementary image containing only the modeled galaxies. To be consistent in the photometry of the extended galaxies we do not use the models. We constructed a new image containing the original pixels (background subtracted) of the removed galaxies. However, to minimize the effect of the projected objects, the intensities of the pixels in which these fell were in fact replaced by the intensities of the models in the same pixels. In practice, this was done constructing a new mask of the objects contained in the background-subtracted image without large objects using SExtractor. Then, the final image was computed following these criteria for its pixels:

- If the pixel did not belong to an object (value in the mask equal to zero) then the background-subtracted image value was kept that corresponded to the original pixel of the galaxy.

- If the pixel indeed belonged to an object (value in the mask greater than zero) then the value in the model was taken instead of the value in the original image.

- If the pixel fell in an interchip region then the value from the model was used if available. In this way, we were able to improve the photometry of large galaxies with large regions lost in the interchip regions.

Of course, for construction, the image of the large galaxies also lacks the bright stars.

This procedure was done in both bands $(V, B)$ removing, of course, the same objects.

\section{Appendix B: Image simulations}

One of the methods most commonly used to check the reliability of a procedure of star/galaxy classification is to test the procedure using images in which synthetic objects have been added. Of course, this method relies upon the idea that the artificial objects are similar enough (from the point of view of the classifying

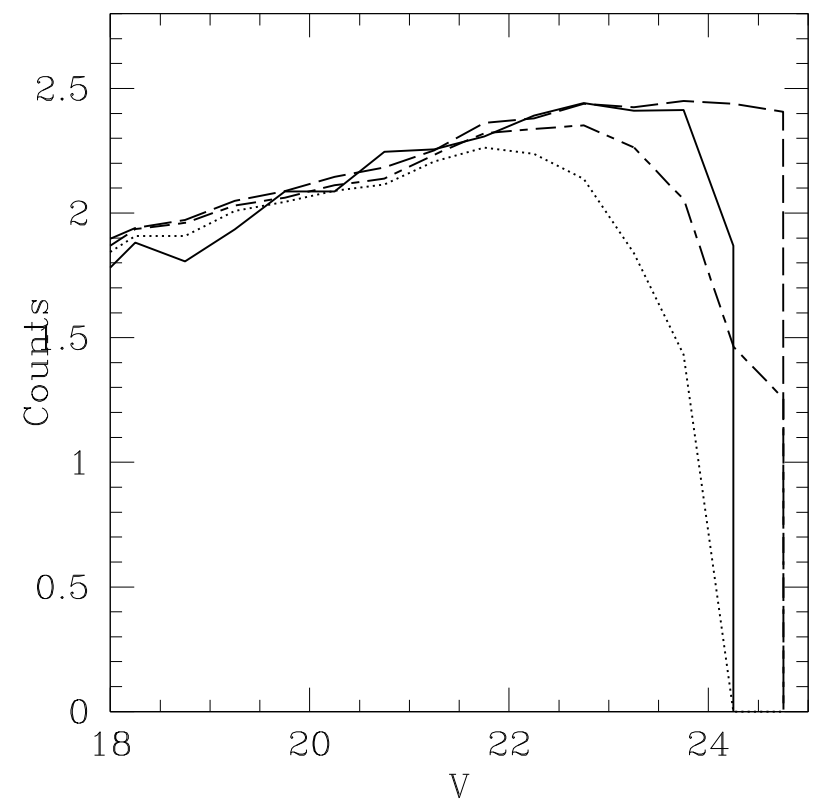

Fig. A.2. Comparison of the real counts (continuous line) with that coming from the simulations. Long dashes line: input distribution of the simulated stars taken from Besançon models. Dotted line: simulated stars classified as stars. Long-short dashed line: simulated stars detected.

program) to the real objects so that one can extrapolate the results obtained from the simulations (from which the input and the output are known) to the real objects.

We followed this method using the tasks of the IRAF's package artdata. We made catalogs of galaxies and stars to build the artificial images that were added to the real images. In this way we could test also the effects of the variations of the background and other real conditions that are difficult or impossible to simulate. The effects of crowding coming from adding more objects to real images were measured to produce less than $10 \%$ of the lost objects in the most crowded image. Then we proceeded with these images as with the real ones. The resulting parameters from SExtractor were compared between real and synthetic objects, showing that they were at first sight quite similar, giving us confidence that the extrapolation from simulations to real objects could be done. However, the comparison of the final counts from the real and the simulated objects made us distrust the simulations, especially that of the faint stars. The reason for this conclusion is illustrated in Fig. A.2. This figure shows the star counts from the original catalogs of WINGS (continuous line) and from the simulations. For simulated stars three lines have been plotted. The long dashed one represents the input catalog and the numbers are taken from the models of the Galaxy from Besançon. The long-short dashed line represents the counts of detected simulated stars. Finally, the dotted line shows the counts of the simulated stars classified as stars, which should be the counts to be compared with the data from WINGS' stars catalog (continuous line). From this figure it can be seen that, at the faintest magnitudes, it was easier for SExtractor to distinguish a real star than a simulated one, violating the initial premise that simulated objects are similar to real ones.

In the construction of the simulated stars we took bright but not saturated stars to have a well sampled point spread function (PSF) even at the wings. So, one expects simulated stars to be more concentrated than real ones and then easier to be classified as stars by SExtractor, which is the opposite of what was 
found. The doubts became greater when simulations were done using two different PSFs, one for bright stars computed from a bright start and another one for faint stars computed from a fainter star. In this case, the fraction of faint simulated stars identified as stars by SExtractor increased. Therefore, the simulations turn out to be too dependent on the input.

As this seemed to be a problem of the mkobjects task we tried with the addstars feature of the daophot package to create the synthetic stars. Since this is a package made to study stars we expected a better treatment of the simulations. However, the results were similar.

The origin of the difficulties is not clear. Probably, small variations of the local conditions where objects are added produce large effects in faint simulated objects.

All these results convinced us not to use the results of the simulations to measure the reliability of our star/galaxy classification at faint magnitudes. However, we did not find such problematic behavior at bright magnitudes. Figure A.2 shows that the problems of detection of simulated stars (before any classification as galaxy or star) start above $V \sim 22$ when the detection rate drops (see Fig. 5). For these reason we decided to still rely on the simulations to estimate the photometric errors and the detection rates since, unfortunately, there is no better procedure to estimate such quantities.

\section{Appendix C: Comparison with WFPC2 @ HST image}

To check the completeness estimations done with simulations we performed a comparison using data from the Hubble Space Telescope. We downloaded the images of the BCG of A1795 taken with the WFPC2 from the HST archive ${ }^{19}$. We chose the $F 555 W$ filter which was the one that best matches the $V$ band images that we used to construct our catalogs. From the mosaic of the WFPC2 we removed the PC chip in which the $\mathrm{BCG}$ was located since this produced problems for SExtractor and we were interested in knowing the completeness at faint magnitudes. After that, SExtractor was run on the image and the resulting catalog was matched and compared with the WINGS catalog. We performed the matching against the global WINGS catalogs, i.e. including stars, galaxies and objects of unknown classification. Figure C.1 (upper panel) shows the completeness computed for the A1795 field using the simulations (continuous line) and the completeness compared with the HST data. We also include the errors in the computation of these data since the area is quite small and therefore the number of detected objects (lower panel) is also quite low.

Although the HST image is much sharper than our groundbased image, it is not much deeper and it also shows problems of completeness in the range of comparison (lower panel of Fig. C.1). This and other issues in the matching procedure (such as pairs of objects that are not resolved in the WINGS image) introduce uncertainties in the comparison so this should be considered as a complementary check to the completeness computed using the simulations.

\section{Appendix D: Additional information}

In addition to the data of the single objects found in each field we include several tables containing information about observa-

\footnotetext{
${ }^{19}$ Based on observations made with the NASA/ESA Hubble Space Telescope, obtained from the data archive at the Space Telescope Science Institute. STScI is operated by the Association of Universities for Research in Astronomy, Inc. under NASA contract NAS 5-26555.
}

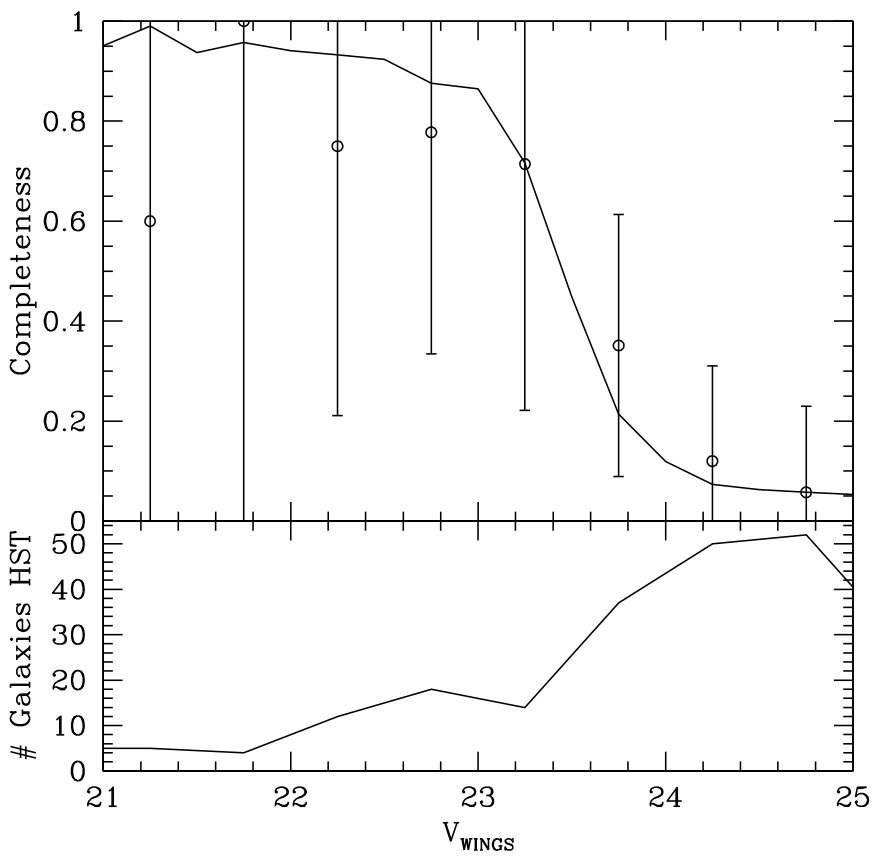

Fig. C.1. Comparison of the completeness of the WINGS catalog of the field of A1795. Upper panel: the continuous line shows the completeness computed from simulations while the dots with the error bars show the completeness when comparing from HST data. The error bars are constructing using $1 \sigma$ Poisson errors. Lower panel: number of objects detected in the HST image. Since the area is quite small the total numbers are also small producing the large uncertainties in the computation of the completeness.

tional features and peculiarities of either the single clusters or the fields on which they are projected.

Position, redshift, Abell richness, Bautz-Morgan type, X-ray luminosity and galactic extinction of the whole WINGS sample can be found in Table 5 of Paper I.

In Table D.1 we summarize the conversion factors used for this work from CCD related units (pixels) to angular unit (arcsecs) and from these to linear units (kpc) at the redshift of the target cluster using a cosmological model with parameters $H_{0}=75 \mathrm{~km} \mathrm{~s}^{-1} \mathrm{Mpc}^{-1}, \Omega_{\mathrm{M}}=0.3$ and $\Omega_{\Lambda}=0.7$. We have also included the effective total area of each image which is the real area used to make the catalogs which is slightly smaller than the total field of view (for WFC@INT images, the effective area is $\sim 90 \%$ of the total field of view while for WFI@ESO this value is $\sim 95 \%$ ). We report the angular sizes of the apertures of $R=2 \mathrm{kpc}$, $5 \mathrm{kpc}$ and $10 \mathrm{kpc}$ used to construct our catalogs.

In Table D. 2 we list the detection limits in surface brightness, or surface brightness thresholds, $\left(\mu_{V}\right.$ (Threshold)) as well as the $V$ band magnitude at which the detection rate goes down to $90 \%, 75 \%$ and $50 \%$. These last values are average values obtained from the simulations.

Table D. 3 reports the positions of the brightest cluster galaxies. In most cases we preferred the coordinates of the peak of the emission instead of the coordinates of the barycenter because the latter are more affected by irregularities in the outer isophotes.

Finally, Table D.4 lists comments or issues about the clusters and the fields which we find interesting or useful when working with our catalogs. 
Table D.1. Useful parameters of the WINGS' clusters sample.

\begin{tabular}{|c|c|c|c|c|c|c|c|c|c|}
\hline \multirow[t]{2}{*}{ Cluster } & \multirow[t]{2}{*}{$\overline{\text { Redshift }}$} & \multirow[t]{2}{*}{ 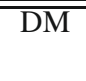 } & \multicolumn{2}{|c|}{ Plate scale } & & Total area & \multicolumn{3}{|c|}{$R_{\text {Aper }}$ in $\operatorname{arcsec}$} \\
\hline & & & arcsec/pixel & $\mathrm{kpc} / \operatorname{arcsec}$ & kpc/pixel $\mathrm{deg}^{2}$ & $\mathrm{Mpc}^{2}$ & $2 \mathrm{kpc}$ & $5 \mathrm{kpc}$ & $10 \mathrm{kpc}$ \\
\hline A85 & 0.0551 & 36.79 & 0.333 & 0.996 & $0.3317 \quad 0.2825$ & 3.632 & 4.23 & 10.57 & 21.15 \\
\hline A119 & 0.0442 & 36.30 & 0.333 & 0.811 & $\begin{array}{lll}0.2701 & 0.2825\end{array}$ & 2.409 & 4.91 & 12.28 & 24.55 \\
\hline A133 & 0.0566 & 36.85 & 0.238 & 1.022 & $\begin{array}{lll}0.2433 & 0.2839\end{array}$ & 3.845 & 3.69 & 9.22 & 18.43 \\
\hline A147 & 0.0447 & 36.32 & 0.333 & 0.820 & $\begin{array}{lll}0.2730 & 0.2825\end{array}$ & 2.460 & 4.92 & 12.31 & 24.62 \\
\hline A151 & 0.0533 & 36.71 & 0.333 & 0.966 & $\begin{array}{lll}0.3217 & 0.2825\end{array}$ & 3.417 & 4.12 & 10.30 & 20.59 \\
\hline A160 & 0.0447 & 36.32 & 0.333 & 0.820 & $\begin{array}{lll}0.2730 & 0.2825\end{array}$ & 2.460 & 4.93 & 12.33 & 24.65 \\
\hline A168 & 0.0450 & 36.34 & 0.333 & 0.825 & $\begin{array}{lll}0.2747 & 0.2736\end{array}$ & 2.412 & 4.89 & 12.23 & 24.47 \\
\hline A193 & 0.0486 & 36.50 & 0.333 & 0.886 & $\begin{array}{lll}0.2950 & 0.2805\end{array}$ & 2.853 & 4.56 & 11.41 & 22.82 \\
\hline A311 & 0.0661 & 37.28 & 0.333 & 1.184 & $\begin{array}{lll}0.3942 & 0.2825\end{array}$ & 5.131 & 3.40 & 8.50 & 16.99 \\
\hline A376 & 0.0484 & 36.49 & 0.333 & 0.883 & $\begin{array}{lll}0.2939 & 0.2825\end{array}$ & 2.852 & 4.50 & 11.24 & 22.49 \\
\hline A500 & 0.0670 & 37.32 & 0.238 & 1.199 & $0.2854 \quad 0.2910$ & 5.423 & 3.34 & 8.34 & 16.68 \\
\hline A548b & 0.0416 & 36.18 & 0.238 & 0.767 & $\begin{array}{lll}0.1825 & 0.2910\end{array}$ & 2.218 & 4.94 & 12.35 & 24.71 \\
\hline A602 & 0.0619 & 37.09 & 0.333 & 1.112 & $\begin{array}{lll}0.3704 & 0.2762\end{array}$ & 4.430 & 3.58 & 8.96 & 17.92 \\
\hline A671 & 0.0502 & 36.57 & 0.333 & 0.913 & $\begin{array}{lll}0.3041 & 0.2762\end{array}$ & 2.986 & 4.37 & 10.93 & 21.86 \\
\hline A754 & 0.0542 & 36.75 & 0.333 & 0.981 & $\begin{array}{lll}0.3268 & 0.2768\end{array}$ & 3.455 & 4.08 & 10.19 & 20.38 \\
\hline A780 & 0.0539 & 36.73 & 0.333 & 0.976 & $\begin{array}{lll}0.3251 & 0.2768\end{array}$ & 3.419 & 3.92 & 9.80 & 19.60 \\
\hline A957x & 0.0436 & 36.27 & 0.333 & 0.801 & $\begin{array}{lll}0.2667 & 0.2762\end{array}$ & 2.297 & 4.87 & 12.17 & 24.35 \\
\hline A970 & 0.0587 & 36.95 & 0.333 & 1.058 & $\begin{array}{lll}0.3523 & 0.2762\end{array}$ & 4.007 & 3.73 & 9.33 & 18.66 \\
\hline A1069 & 0.0650 & 37.23 & 0.333 & 1.165 & $0.388 \quad 0.2762$ & 4.860 & 3.58 & 8.95 & 17.90 \\
\hline A1291 & 0.0527 & 36.68 & 0.333 & 0.956 & $\begin{array}{lll}0.3183 & 0.2762\end{array}$ & 3.271 & 4.18 & 10.46 & 20.92 \\
\hline A1631a & 0.0462 & 36.39 & 0.238 & 0.845 & $0.2012 \quad 0.2874$ & 2.661 & 4.69 & 11.74 & 23.47 \\
\hline A1644 & 0.0473 & 36.44 & 0.238 & 0.864 & $\begin{array}{lll}0.2056 & 0.2874\end{array}$ & 2.780 & 4.61 & 11.53 & 23.06 \\
\hline A1668 & 0.0634 & 37.16 & 0.333 & 1.138 & $\begin{array}{lll}0.3789 & 0.2762\end{array}$ & 4.636 & 3.52 & 8.79 & 17.58 \\
\hline A1736 & 0.0458 & 36.37 & 0.238 & 0.838 & $\begin{array}{lll}0.1995 & 0.2847\end{array}$ & 2.594 & 4.74 & 11.85 & 23.71 \\
\hline A1795 & 0.0625 & 37.12 & 0.333 & 1.122 & $\begin{array}{lll}0.3737 & 0.2762\end{array}$ & 4.509 & 3.58 & 8.95 & 17.90 \\
\hline A1831 & 0.0615 & 37.07 & 0.333 & 1.106 & $\begin{array}{lll}0.3682 & 0.2762\end{array}$ & 4.376 & 3.63 & 9.09 & 18.17 \\
\hline A1983 & 0.0436 & 36.27 & 0.333 & 0.801 & $\begin{array}{lll}0.2667 & 0.2715\end{array}$ & 2.257 & 4.91 & 12.28 & 24.55 \\
\hline A1991 & 0.0587 & 36.95 & 0.333 & 1.058 & $\begin{array}{ll}0.3523 & 0.2762\end{array}$ & 4.007 & 3.79 & 9.47 & 18.93 \\
\hline A2107 & 0.0412 & 36.16 & 0.333 & 0.759 & $\begin{array}{lll}0.2528 & 0.2872\end{array}$ & 2.146 & 5.32 & 13.30 & 26.61 \\
\hline A2124 & 0.0656 & 37.26 & 0.333 & 1.176 & $\begin{array}{lll}0.3916 & 0.2762\end{array}$ & 4.950 & 3.41 & 8.53 & 17.07 \\
\hline A2149 & 0.0679 & 37.36 & 0.333 & 1.215 & $\begin{array}{lll}0.4044 & 0.2762\end{array}$ & 5.281 & 3.31 & 8.28 & 16.56 \\
\hline A2169 & 0.0586 & 36.94 & 0.333 & 1.056 & $\begin{array}{lll}0.3517 & 0.2825\end{array}$ & 4.084 & 3.83 & 9.58 & 19.15 \\
\hline A2256 & 0.0581 & 36.92 & 0.333 & 1.048 & $\begin{array}{lll}0.3489 & 0.2820\end{array}$ & 4.013 & 3.82 & 9.54 & 19.09 \\
\hline A2271 & 0.0576 & 36.90 & 0.333 & 1.039 & $\begin{array}{lll}0.3461 & 0.2768\end{array}$ & 3.874 & 3.80 & 9.50 & 19.00 \\
\hline A2382 & 0.0618 & 37.09 & 0.238 & 1.111 & $0.2643 \quad 0.2916$ & 4.662 & 3.46 & 8.66 & 17.32 \\
\hline A2399 & 0.0579 & 36.91 & 0.238 & 1.044 & $\begin{array}{lll}0.2486 & 0.2843\end{array}$ & 4.018 & 3.81 & 9.53 & 19.06 \\
\hline A2415 & 0.0581 & 36.92 & 0.333 & 1.048 & $\begin{array}{lll}0.3489 & 0.2740\end{array}$ & 3.898 & 3.80 & 9.49 & 18.98 \\
\hline A2457 & 0.0594 & 36.98 & 0.333 & 1.070 & $\begin{array}{lll}0.3563 & 0.2825\end{array}$ & 4.190 & 3.76 & 9.39 & 18.78 \\
\hline A2572a & 0.0403 & 36.13 & 0.333 & 0.745 & $\begin{array}{lll}0.2480 & 0.2825\end{array}$ & 2.031 & 5.19 & 12.98 & 25.97 \\
\hline A2589 & 0.0414 & 36.17 & 0.333 & 0.764 & $\begin{array}{lll}0.2543 & 0.2825\end{array}$ & 2.134 & 5.26 & 13.16 & 26.31 \\
\hline A2593 & 0.0413 & 36.17 & 0.333 & 0.762 & $\begin{array}{lll}0.2537 & 0.2825\end{array}$ & 2.125 & 5.08 & 12.70 & 25.40 \\
\hline A2622 & 0.0620 & 37.10 & 0.333 & 1.114 & $\begin{array}{lll}0.3710 & 0.2825\end{array}$ & 4.544 & 3.63 & 9.07 & 18.15 \\
\hline A2626 & 0.0553 & 36.80 & 0.333 & 1.000 & $\begin{array}{lll}0.3330 & 0.2740\end{array}$ & 3.551 & 3.92 & 9.80 & 19.60 \\
\hline A2657 & 0.0402 & 36.12 & 0.333 & 0.743 & $\begin{array}{lll}0.2475 & 0.2825\end{array}$ & 2.022 & 5.41 & 13.52 & 27.04 \\
\hline A2665 & 0.0556 & 36.81 & 0.333 & 1.005 & $\begin{array}{lll}0.3347 & 0.2825\end{array}$ & 3.699 & 3.94 & 9.85 & 19.70 \\
\hline A2717 & 0.0490 & 36.51 & 0.238 & 0.893 & $\begin{array}{lll}0.2125 & 0.2832\end{array}$ & 2.926 & 4.41 & 11.03 & 22.06 \\
\hline A2734 & 0.0625 & 37.12 & 0.238 & 1.123 & $\begin{array}{lll}0.2672 & 0.2832\end{array}$ & 4.626 & 3.57 & 8.92 & 17.84 \\
\hline A3128 & 0.0599 & 37.00 & 0.238 & 1.078 & $\begin{array}{lll}0.2567 & 0.2916\end{array}$ & 4.394 & 3.76 & 9.41 & 18.81 \\
\hline A3158 & 0.0597 & 36.99 & 0.238 & 1.075 & $\begin{array}{lll}0.2558 & 0.2916\end{array}$ & 4.367 & 3.76 & 9.41 & 18.81 \\
\hline A3164 & 0.0570 & 36.87 & 0.238 & 1.029 & $\begin{array}{lll}0.2449 & 0.2842\end{array}$ & 3.900 & 3.64 & 9.10 & 18.20 \\
\hline A3266 & 0.0589 & 36.96 & 0.238 & 1.061 & $\begin{array}{lll}0.2526 & 0.2916\end{array}$ & 4.257 & 4.05 & 10.14 & 20.27 \\
\hline A3376 & 0.0456 & 36.36 & 0.238 & 0.835 & 0.19870 .2916 & 2.635 & 4.71 & 11.78 & 23.57 \\
\hline A3395 & 0.0506 & 36.59 & 0.238 & 0.920 & $\begin{array}{lll}0.2190 & 0.2910\end{array}$ & 3.192 & 4.42 & 11.05 & 22.10 \\
\hline A3490 & 0.0688 & 37.40 & 0.238 & 1.230 & $0.2927 \quad 0.2874$ & 5.634 & 3.21 & 8.03 & 16.06 \\
\hline A3497 & 0.0677 & 37.35 & 0.238 & 1.211 & $\begin{array}{lll}0.2882 & 0.2874\end{array}$ & 5.463 & 3.65 & 9.13 & 18.26 \\
\hline A3528a & 0.0535 & 36.72 & 0.238 & 0.970 & $0.2307 \quad 0.2803$ & 3.414 & 4.13 & 10.31 & 20.63 \\
\hline A3528b & 0.0535 & 36.72 & 0.238 & 0.970 & $\begin{array}{lll}0.2307 & 0.2847\end{array}$ & 3.468 & 4.13 & 10.31 & 20.63 \\
\hline A3530 & 0.0537 & 36.72 & 0.238 & 0.973 & $\begin{array}{lll}0.2315 & 0.2837\end{array}$ & 3.480 & 4.06 & 10.15 & 20.31 \\
\hline A3532 & 0.0554 & 36.80 & 0.238 & 1.002 & 0.23840 .2890 & 3.758 & 3.99 & 9.97 & 19.93 \\
\hline A3556 & 0.0479 & 36.47 & 0.238 & 0.874 & $\begin{array}{lll}0.2081 & 0.2874\end{array}$ & 2.846 & 4.48 & 11.20 & 22.40 \\
\hline A3558 & 0.0480 & 36.47 & 0.238 & 0.876 & $\begin{array}{lll}0.2085 & 0.2874\end{array}$ & 2.857 & 4.59 & 11.48 & 22.97 \\
\hline A3560 & 0.0489 & 36.51 & 0.238 & 0.891 & $\begin{array}{lll}0.2121 & 0.2874\end{array}$ & 2.958 & 4.66 & 11.64 & 23.29 \\
\hline
\end{tabular}


Table D.1. continued.

\begin{tabular}{|c|c|c|c|c|c|c|c|c|c|}
\hline \multirow[t]{2}{*}{$\overline{\bar{C} \text { Cluster }}$} & \multirow[t]{2}{*}{ Redshift } & \multirow[t]{2}{*}{$\overline{\mathrm{DM}}$} & \multicolumn{3}{|c|}{ Plate scale } & Total area & \multicolumn{3}{|c|}{$R_{\text {Aper }}$ in arcsec } \\
\hline & & & arcsec/pixel & $\mathrm{kpc} / \operatorname{arcsec}$ & kpc/pixel $\mathrm{deg}^{2}$ & $\mathrm{Mpc}^{2}$ & $2 \mathrm{kpc}$ & $5 \mathrm{kpc}$ & $10 \mathrm{kpc}$ \\
\hline A3562 & 0.0490 & 36.51 & 0.238 & 0.893 & $\begin{array}{ll}0.2125 & 0.2828\end{array}$ & 2.922 & 4.38 & 10.95 & 21.90 \\
\hline A3667 & 0.0556 & 36.81 & 0.238 & 1.005 & $\begin{array}{lll}0.2392 & 0.2822\end{array}$ & 3.696 & 4.16 & 10.41 & 20.81 \\
\hline A3716 & 0.0462 & 36.39 & 0.238 & 0.845 & $0.2012 \quad 0.2839$ & 2.629 & 4.87 & 12.17 & 24.35 \\
\hline A3809 & 0.0620 & 37.10 & 0.238 & 1.114 & $0.2652 \quad 0.2838$ & 4.566 & 3.53 & 8.83 & 17.65 \\
\hline A3880 & 0.0584 & 36.93 & 0.238 & 1.053 & $\begin{array}{lll}0.2506 & 0.2832\end{array}$ & 4.069 & 3.89 & 9.72 & 19.44 \\
\hline A4059 & 0.0475 & 36.45 & 0.238 & 0.867 & $0.2064 \quad 0.2832$ & 2.761 & 4.57 & 11.42 & 22.83 \\
\hline IIZW108 & 0.0493 & 36.53 & 0.333 & 0.899 & $\begin{array}{lll}0.2993 & 0.2825\end{array}$ & 2.958 & 4.54 & 11.35 & 22.70 \\
\hline MKW3s & 0.0450 & 36.34 & 0.333 & 0.825 & $\begin{array}{lll}0.2747 & 0.2762\end{array}$ & 2.435 & 4.82 & 12.05 & 24.10 \\
\hline RX0058 & 0.0470 & 36.43 & 0.333 & 0.859 & $\begin{array}{lll}0.2860 & 0.2825\end{array}$ & 2.700 & 4.66 & 11.64 & 23.29 \\
\hline RX1022 & 0.0491 & 36.52 & 0.333 & 0.895 & 0.29790 .2762 & 2.865 & 4.13 & 10.33 & 20.67 \\
\hline RX1740 & 0.0430 & 36.25 & 0.333 & 0.791 & $\begin{array}{lll}0.2633 & 0.2768\end{array}$ & 2.243 & 5.06 & 12.65 & 25.29 \\
\hline Z1261 & 0.0644 & 37.20 & 0.333 & 1.155 & $\begin{array}{lll}0.3846 & 0.2825\end{array}$ & 4.884 & 3.46 & 8.66 & 17.32 \\
\hline Z2844 & 0.0500 & 36.56 & 0.333 & 0.910 & $0.3030 \quad 0.2762$ & 2.964 & 4.40 & 10.99 & 21.98 \\
\hline Z8338 & 0.0473 & 36.44 & 0.333 & 0.864 & $\begin{array}{lll}0.2877 & 0.2768\end{array}$ & 2.678 & 4.63 & 11.57 & 23.15 \\
\hline Z8852 & 0.0400 & 36.11 & 0.333 & 0.740 & $0.2463 \quad 0.2825$ & 2.003 & 5.41 & 13.52 & 27.04 \\
\hline
\end{tabular}

DM: distance modulus.

Total area: this is the effective area used to construct the catalogs. To compute it the interchip regions have been subtracted.

$D_{\text {Aper }}$ : conversion of the physical apertures to angular sizes.

Table D.2. Completeness limits and surface brightness detection limits.

\begin{tabular}{|c|c|c|c|c|c|c|c|c|c|}
\hline \multirow[t]{2}{*}{ Cluster } & \multicolumn{3}{|c|}{$\bar{V} \overline{\text { detection rate limits }}$} & \multirow{2}{*}{$\begin{array}{c}\mu_{V}(\text { Threshold }) \\
\text { mag/arcsec }\end{array}$} & \multirow[t]{2}{*}{ Cluster } & \multicolumn{3}{|c|}{$V$ detection rate limits } & \multirow{2}{*}{ 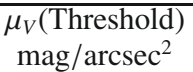 } \\
\hline & $\geq 90 \%$ & $\geq 75 \%$ & $\geq 50 \%$ & & & $\geq 90 \%$ & $\geq 75 \%$ & $\geq 50 \%$ & \\
\hline A 85 & 22.6 & 22.9 & 23.2 & 25.85 & A2589 & 22.4 & 23.1 & 23.5 & 25.89 \\
\hline A119 & 22.6 & 22.9 & 23.2 & 25.92 & A2593 & 22.4 & 23.1 & 23.4 & 25.87 \\
\hline A133 & 22.3 & 22.8 & 23.1 & 25.56 & A2622 & 21.6 & 22.9 & 23.3 & 25.93 \\
\hline A147 & 22.4 & 23.0 & 23.3 & 25.89 & A2626 & 21.9 & 22.8 & 23.2 & 25.98 \\
\hline A151 & 22.2 & 22.8 & 23.2 & 25.73 & A2657 & 22.4 & 22.9 & 23.3 & 25.93 \\
\hline A160 & 22.5 & 23.0 & 23.3 & 25.85 & A2665 & 22.6 & 23.1 & 23.4 & 25.88 \\
\hline A168 & 22.6 & 23.1 & 23.4 & 25.90 & A2717 & 22.4 & 22.8 & 23.2 & 25.12 \\
\hline A193 & 22.7 & 23.1 & 23.4 & 25.86 & A2734 & 22.5 & 23.0 & 23.3 & 25.52 \\
\hline A311 & 22.6 & 23.1 & 23.4 & 26.00 & A3128 & 22.1 & 22.7 & 23.2 & 25.54 \\
\hline A376 & 21.4 & 22.7 & 23.2 & 25.89 & A3158 & 21.4 & 23.0 & 23.4 & 25.61 \\
\hline A500 & 22.8 & 23.2 & 23.5 & 25.53 & A3164 & 21.5 & 22.8 & 23.2 & 25.71 \\
\hline A548b & 21.8 & 22.9 & 23.3 & 25.42 & A3266 & 21.3 & 22.9 & 23.3 & 25.46 \\
\hline A602 & 22.0 & 22.7 & 23.1 & 25.69 & A3376 & 20.9 & 22.7 & 23.2 & 25.39 \\
\hline A671 & 22.1 & 22.7 & 23.1 & 25.75 & A3395 & 21.3 & 23.0 & 23.3 & 25.35 \\
\hline A754 & 20.8 & 22.6 & 23.0 & 25.60 & A3490 & 21.2 & 22.6 & 23.1 & 25.38 \\
\hline A780 & 20.7 & 21.6 & 22.2 & 25.29 & A3497 & 22.2 & 23.2 & 23.7 & 25.60 \\
\hline A957x & 22.0 & 22.6 & 22.9 & 25.64 & A3528a & 22.6 & 23.1 & 23.4 & 25.40 \\
\hline A970 & 21.7 & 22.6 & 22.8 & 25.57 & A3528b & 22.3 & 22.7 & 23.1 & 25.01 \\
\hline A1069 & 22.1 & 22.7 & 23.1 & 25.68 & A3530 & 22.0 & 22.9 & 23.3 & 25.40 \\
\hline A1291 & 22.2 & 22.7 & 23.1 & 25.74 & A3532 & 21.2 & 23.1 & 23.5 & 25.51 \\
\hline A1631a & 22.5 & 23.1 & 23.5 & 25.39 & A3556 & 21.4 & 23.1 & 23.5 & 25.56 \\
\hline A1644 & 22.7 & 23.2 & 23.5 & 25.42 & A3558 & 21.8 & 23.0 & 23.5 & 25.56 \\
\hline A1668 & 21.9 & 23.1 & 23.4 & 26.12 & A3560 & 21.1 & 23.1 & 23.5 & 25.59 \\
\hline A1736 & 22.1 & 23.1 & 23.6 & 25.49 & A3562 & 19.8 & 21.6 & 22.0 & 25.47 \\
\hline A1795 & 22.6 & 23.1 & 23.4 & 25.96 & A3667 & 19.7 & 22.1 & 22.9 & 25.56 \\
\hline A1831 & 22.5 & 23.0 & 23.3 & 25.83 & A3716 & 21.3 & 22.6 & 23.0 & 25.53 \\
\hline A1983 & 22.7 & 23.1 & 23.5 & 26.00 & A3809 & 22.5 & 23.1 & 23.5 & 25.54 \\
\hline A1991 & 22.7 & 23.1 & 23.4 & 26.00 & A3880 & 22.6 & 23.0 & 23.3 & 25.52 \\
\hline A2107 & 22.5 & 22.9 & 23.2 & 25.78 & A4059 & 22.4 & 23.0 & 23.3 & 25.49 \\
\hline A2124 & 22.6 & 23.0 & 23.3 & 25.84 & IIZW108 & 19.8 & 22.1 & 23.0 & 25.86 \\
\hline A2149 & 21.6 & 22.0 & 22.3 & 24.65 & MKW3s & 21.6 & 22.1 & 22.4 & 24.80 \\
\hline A2169 & 22.4 & 23.0 & 23.3 & 25.85 & RX0058 & 22.5 & 23.1 & 23.5 & 26.03 \\
\hline A2256 & 20.7 & 22.6 & 23.1 & 25.77 & RX1022 & 22.7 & 23.1 & 23.3 & 25.75 \\
\hline A2271 & 21.5 & 21.9 & 22.2 & 24.79 & RX1740 & 21.1 & 22.8 & 23.3 & 26.01 \\
\hline A2382 & 22.2 & 22.8 & 23.2 & 25.50 & Z1261 & 20.8 & 22.7 & 23.2 & 25.82 \\
\hline A2399 & 22.1 & 22.7 & 23.2 & 25.48 & Z2844 & 22.6 & 22.9 & 23.3 & 25.70 \\
\hline A2415 & 22.1 & 22.8 & 23.2 & 25.75 & Z8338 & 21.4 & 22.9 & 23.4 & 26.03 \\
\hline A2457 & 22.1 & 22.9 & 23.2 & 25.82 & Z8852 & 21.7 & 22.7 & 23.2 & 25.83 \\
\hline A2572a & 21.9 & 23.0 & 23.4 & 25.93 & & & & & \\
\hline
\end{tabular}


J. Varela et al.: WINGS II: deep optical catalogs, Online Material p 6

Table D.3. Coordinates of the emission peak of the brightest cluster galaxies.

\begin{tabular}{|c|c|c|c|c|c|}
\hline \multirow[t]{2}{*}{ Cluster } & \multicolumn{2}{|c|}{ Equat.Coordinates (J2000.0) } & \multirow[t]{2}{*}{ Cluster } & \multicolumn{2}{|c|}{ Equat.Coordinates (J2000.0) } \\
\hline & $\alpha$ & $\sigma$ & & & $\delta$ \\
\hline A 85 & $0: 41: 50.5$ & $-9: 18: 11.5$ & A2589 & $23: 23: 57.5$ & $16: 46: 38.3$ \\
\hline A119 & $0: 56: 16.1$ & $-1: 15: 19.1$ & A2593 & $23: 24: 20.1$ & $14: 38: 49.8$ \\
\hline A133 & 1:02:41.7 & $-21: 52: 55.5$ & A2622 & $23: 35: 01.5$ & $27: 22: 21.0$ \\
\hline A147 & 1:08:12.0 & $2: 11: 38.2$ & A2626 & $23: 36: 30.5$ & 21:08:47.4 \\
\hline A151 & 1:08:51.1 & $-15: 24: 23.1$ & A2657 & $23: 44: 57.4$ & $9: 11: 35.3$ \\
\hline A160 & 1:12:59.6 & $15: 29: 28.9$ & A2665 & 23:50:50.6 & $6: 08: 58.9$ \\
\hline A168 & 1:14:57.6 & $0: 25: 51.2$ & A2717 & 0:03:13.0 & $-35: 56: 13.3$ \\
\hline A193 & $1: 25: 07.6$ & $8: 41: 57.2$ & A2734 & $0: 11: 21.6$ & $-28: 51: 15.6$ \\
\hline A311 & 2:09:28.4 & $19: 46: 36.2$ & A 3128 & $3: 29: 50.6$ & $-52: 34: 46.8$ \\
\hline A376 & 2:46:03.9 & $36: 54: 19.2$ & A3158 & $3: 42: 53.0$ & $-53: 37: 52.6$ \\
\hline A500 & $4: 38: 52.5$ & $-22: 06: 39.0$ & A3164 & $3: 45: 25.8$ & $-56: 59: 02.0$ \\
\hline A548b & $5: 45: 29.6$ & $-25: 55: 56.8$ & A3266 & $4: 31: 13.3$ & $-61: 27: 12.0$ \\
\hline A602 & $7: 53: 26.6$ & $29: 21: 34.5$ & A3376 & 6:00:41.1 & $-40: 02: 40.4$ \\
\hline A671 & $8: 28: 31.7$ & $30: 25: 53.1$ & A3395 & $6: 27: 36.3$ & $-54: 26: 57.9$ \\
\hline A754 & 9:08:32.4 & $-9: 37: 47.4$ & A3490 & $11: 45: 20.2$ & $-34: 25: 59.4$ \\
\hline A780 & 9:18:05.7 & $-12: 05: 43.4$ & A3497 & 11:59:46.3 & $-31: 31: 41.6$ \\
\hline A957x & 10:13:38.3 & $-0: 55: 31.3$ & A3528a & $12: 54: 41.0$ & $-29: 13: 39.5$ \\
\hline A970 & $10: 17: 25.7$ & $-10: 41: 20.2$ & A3528b & $12: 54: 22.2$ & $-29: 00: 46.8$ \\
\hline A1069 & 10:39:43.4 & $-8: 41: 12.4$ & A3530 & $12: 55: 36.0$ & $-30: 20: 51.4$ \\
\hline A1291 & 11:32:23.2 & 55:58:03.0 & A3532 & $12: 57: 22.0$ & $-30: 21: 49.1$ \\
\hline A1631a & 12:52:52.6 & $-15: 24: 47.8$ & A3556 & 13:24:06.7 & $-31: 40: 11.6$ \\
\hline A1644 & 12:57:11.6 & $-17: 24: 34.0$ & A3558 & $13: 27: 56.8$ & $-31: 29: 44.0$ \\
\hline A1668 & 13:03:46.6 & $19: 16: 17.4$ & A3560 & $13: 31: 53.5$ & $-33: 14: 03.1$ \\
\hline A1736 & $13: 27: 28.0$ & $-27: 19: 29.2$ & A3562 & 13:33:34.7 & $-31: 40: 20.5$ \\
\hline A1795 & 13:48:52.5 & $26: 35: 34.6$ & A3667 & $20: 12: 27.3$ & $-56: 49: 36.4$ \\
\hline A1831 & 13:59:15.1 & $27: 58: 34.5$ & A3716 & 20:51:19.9 & $-52: 38: 10.5$ \\
\hline A1983 & $14: 52: 55.3$ & $16: 42: 10.6$ & A3809 & 21:46:59.1 & $-43: 53: 56.2$ \\
\hline A1991 & 14:54:31.5 & $18: 38: 32.9$ & A3880 & $22: 27: 54.4$ & $-30: 34: 31.9$ \\
\hline A2107 & 15:39:39.0 & $21: 46: 58.0$ & A4059 & 23:57:00.7 & $-34: 45: 32.9$ \\
\hline A2124 & $15: 44: 59.0$ & $36: 06: 33.9$ & IIZW108 & 21:13:55.9 & $2: 33: 55.4$ \\
\hline A2149 & 16:01:28.1 & $53: 56: 50.4$ & MKW3s & $15: 21: 51.9$ & $7: 42: 32.1$ \\
\hline A2169 & 16:13:58.1 & $49: 11: 22.4$ & RX0058 & $0: 58: 22.6$ & $26: 51: 59.0$ \\
\hline A2256 & 17:04:27.2 & $78: 38: 25.4$ & RX1022 & $10: 22: 10.0$ & $38: 31: 23.9$ \\
\hline A2271 & 17:18:16.7 & 78:01:06.2 & RX1740 & $17: 40: 32.1$ & $35: 38: 46.1$ \\
\hline A2382 & 21:51:55.6 & $-15: 42: 21.3$ & Z1261 & $7: 16: 41.2$ & $53: 23: 09.5$ \\
\hline A2399 & 21:57:01.7 & $-7: 50: 22.0$ & Z2844 & 10:02:36.5 & $32: 42: 24.3$ \\
\hline A2415 & 22:05:38.6 & $-5: 35: 32.1$ & Z8338 & 18:11:05.2 & $49: 54: 33.7$ \\
\hline A2457 & 22:35:40.8 & $1: 29: 05.9$ & Z8852 & 23:10:22.4 & $7: 34: 50.6$ \\
\hline A2572a & 23:17:12.0 & $18: 42: 04.7$ & & & \\
\hline
\end{tabular}


Table D.4. Remarks about the individual fields.

\begin{tabular}{|c|c|}
\hline Cluster & Notes \\
\hline \multicolumn{2}{|l|}{ A85 } \\
\hline \multicolumn{2}{|l|}{ A119 } \\
\hline A133 & Two very bright stars in $(\alpha, \delta)_{J 2000.0}=\left(15.61552^{\circ},-21.60940^{\circ}\right)$ and $\left(15.54687^{\circ},-21.60804^{\circ}\right)$ \\
\hline A147 & Very bright star in $\left(16.99828^{\circ}, 1.99262^{\circ}\right)$ \\
\hline A151 & Background structure at $z \sim 0.096$ \\
\hline A160 & Background group at $z \sim 0.060$ \\
\hline A168 & Merger of a spiral dominated cluster and a cD dominated one \\
\hline \multicolumn{2}{|l|}{ A193 } \\
\hline \multicolumn{2}{|l|}{ A311 } \\
\hline \multirow[t]{2}{*}{ A376 } & Strong spatial variation of the PSF along the declination direction \\
\hline & Bright star in $(\alpha, \delta)_{J 2000.0}=\left(41.13167^{\circ}, 36.76294^{\circ}\right)$ \\
\hline \multicolumn{2}{|r|}{ - } \\
\hline A548b & Interacting central pair of galaxies \\
\hline A602 & Cluster with two similar dominant galaxies \\
\hline \multicolumn{2}{|r|}{ ( } \\
\hline A754 & Two bright stars at $\left(137.16750^{\circ},-9.38406^{\circ}\right)$ and $\left(136.95036^{\circ},-9.85370^{\circ}\right)$ \\
\hline \multirow[t]{3}{*}{ A780 } & Bright stars at $\left(139.52435^{\circ},-12.319313^{\circ}\right)$ and $\left(139.31748^{\circ},-12.00786^{\circ}\right)$ \\
\hline & BCG contaminated by two stars that are at $\sim 1$ arcmin \\
\hline & High contamination from a star outside of the field of view. \\
\hline A957x & Very bright star at $\left(153.63937^{\circ},-1.11778^{\circ}\right)$ \\
\hline \multicolumn{2}{|r|}{ 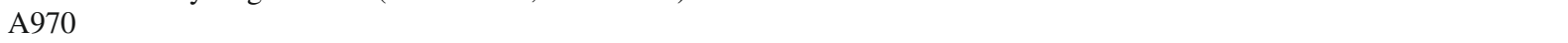 } \\
\hline \multicolumn{2}{|l|}{ A1069 } \\
\hline A1291 & Bright star in $\left(173.08422^{\circ}, 56.09649^{\circ}\right)$ \\
\hline \multirow[t]{2}{*}{ A1631a } & Contaminated by a foreground group at $z \sim 0.0144$ \\
\hline & The brightest galaxy in the center of the field is a foreground galaxy \\
\hline \multirow[t]{2}{*}{ A1644 } & Merging between two clusters \\
\hline & Bright star close to the center $\left(194.39669^{\circ},-17.39507^{\circ}\right)$ \\
\hline \multicolumn{2}{|r|}{ - } \\
\hline A1736 & $\begin{array}{l}\text { Cluster without a clear center and with two dominant galaxies quite far away one from the other }\left(\gtrsim 14^{\prime} \gtrsim 700 \mathrm{kpc}\right) \text {. } \\
\text { Possible merger? }\end{array}$ \\
\hline \multirow{2}{*}{ A1795 } & Bright star at $\left(207.01130^{\circ}, 26.51543^{\circ}\right)$ \\
\hline & Bright star at $\left(207.20086^{\circ}, 26.61439^{\circ}\right)$ at only $\sim 1.67$ of the BCG \\
\hline \multicolumn{2}{|r|}{ - } \\
\hline A1983 & Two dominant galaxies \\
\hline A1991 & Bright star at $\left(223.49666^{\circ}, 18.49984^{\circ}\right)$ \\
\hline A2107 & Only field observed using high-dithering technique \\
\hline A2124 & \\
\hline A2149 & Very bright star at $\left(240.10335^{\circ}, 53.96715^{\circ}\right)$ \\
\hline A2169 & Bright spike near the edge of one chip from $(4050,1)$ to $(4050, \sim 100)$ \\
\hline A2256 & Field with a high star density \\
\hline & Bright star at $\left(255.89125^{\circ}, 78.630028^{\circ}\right)$ \\
\hline A2271 & Bright star in $\left(257.09583^{\circ}, 78.828472^{\circ}\right)$ \\
\hline A2382 & Bright star at $\left(328.15959^{\circ},-15.80787^{\circ}\right)$ \\
\hline A2399 & The brightest galaxy in the field is indeed a foreground $(z=0.017)$ spiral galaxy \\
\hline A2415 & Bright star at $\left(331.52216^{\circ},-5.35782^{\circ}\right)$ \\
\hline A2457 & Contaminated by a galactic nebula \\
\hline A2572a & Central pair of galaxies in interaction \\
\hline & Bright star at $\left(349.47527^{\circ}, 18.70290^{\circ}\right)$ \\
\hline A2589 & Background group at $z \sim 0.17$ \\
\hline A2593 & Bright star at $\left(351.09915^{\circ}, 14.47857^{\circ}\right)$ \\
\hline A2622 & \\
\hline A2626 & Contaminated by A2625 $(z=0.0609)$ \\
\hline A2657 & Field contaminated by a galactic nebula \\
\hline A2665 & Bright stars at $\left(357.65235^{\circ}, 6.01907^{\circ}\right)$ \\
\hline A2717 & Bright star at $\left(0.85301^{\circ},-35.96565^{\circ}\right)$ close to the BCG $\left(\sim 2{ }^{\prime} .9\right)$ \\
\hline A2734 & \\
\hline A 3128 & Bright star at $\left(53.00461^{\circ},-52.47426^{\circ}\right)$ \\
\hline A 3158 & \\
\hline A3164 & BCG lost in interchip region!! \\
\hline A3266 & Bright star at $\left(67.97359^{\circ},-61.38483^{\circ}\right)$ \\
\hline A3376 & Bright star at $\left(90.15115^{\circ},-39.95171^{\circ}\right)$ \\
\hline A3395 & \\
\hline A3490 & \\
\hline A3497 & About half of the BCG falls in an interchip region \\
\hline A3528a & Double system together with A3528b \\
\hline & Member of a group of clusters with A3528a, A3530 and A3532 \\
\hline & Bright stars at $\left(193.55279^{\circ},-29.40419^{\circ}\right)$ and $\left(193.47205^{\circ},-29.30771^{\circ}\right)$ \\
\hline
\end{tabular}


Table D.4. continued.

\begin{tabular}{|c|c|}
\hline Cluster & Notes \\
\hline \multirow[t]{3}{*}{$\mathrm{A} 3528 \mathrm{~b}$} & Only $460 \mathrm{~s}$ of total exposure time in $V$ and $180 \mathrm{~s}$ in $B$ \\
\hline & Double system together with A3528a \\
\hline & Member of a group of clusters with A3528b, A3530 and A3532 \\
\hline \multirow[t]{3}{*}{ A 3530} & Member of a group of clusters with A3528a, A3528b and A3532 \\
\hline & BCG in interaction with a close galaxy \\
\hline & Bright star at $\left(193.88556^{\circ},-30.06425^{\circ}\right)$ \\
\hline A3532 & Member of a group of clusters with A3528a, A3528b and A3530 \\
\hline A3556 & Cluster with two dominant galaxies \\
\hline A3558 & Bright stars at $\left(202.05404^{\circ},-31.55222^{\circ}\right)$ and $\left(201.64741^{\circ},-31.45133^{\circ}\right)$ \\
\hline A 3560 & $\begin{array}{l}\text { The central galaxy of the field which is also the brightest galaxy in the field is a foreground galaxy }(z=0.0124) \\
\text { Bright star at }\left(203.06308^{\circ},-33.24893^{\circ}\right)\end{array}$ \\
\hline A 3562 & $\begin{array}{l}\text { Total exposure times of } 180 \mathrm{~s} \text { in each filter } \\
\text { Very bad seeing }\left(F W H M_{*}(V) \sim 2^{\prime \prime} 38\right)\end{array}$ \\
\hline A 3667 & Bright star at $\left(303.24178^{\circ},-56.84679^{\circ}\right)$ \\
\hline \multirow[t]{3}{*}{ A 3716} & Brightest galaxy in the field is a background galaxy $(z=0.0557)$ \\
\hline & Two bright central galaxies maybe in interaction \\
\hline & Bright star at $\left(312.81216^{\circ},-52.62427^{\circ}\right)$ \\
\hline A3809 & Bright star at $\left(326.41697^{\circ},-44.13961^{\circ}\right)$ \\
\hline A3880 & Bright star at $\left(336.96038^{\circ},-30.60259^{\circ}\right)$ at $\sim 1^{\prime} .82$ from the BCG \\
\hline \multicolumn{2}{|r|}{ o } \\
\hline \multirow[t]{2}{*}{ IIZW108 } & BCG in clear interaction \\
\hline & Very bright star at $\left(318.18880^{\circ}, 2.64289^{\circ}\right)$ \\
\hline MKW3s & Two bright stars at $\left(230.21682^{\circ}, 7.68269^{\circ}\right)$ and $\left(230.59067^{\circ}, 7.81603^{\circ}\right)$, respectively \\
\hline \multirow[t]{3}{*}{$\mathrm{RX} 58$} & BCG with two nuclei separated by $\sim 7^{\prime \prime}$ \\
\hline & In the catalog they are considered as two objects \\
\hline & Two bright stars $\left(14.61869^{\circ}, 26.78588^{\circ}\right)$ and $\left(14.66231^{\circ}, 26.85845^{\circ}\right)$ both closer than 5 arcmin to the BCG \\
\hline RX1022 & Problem with photometry found, probably extinction by clouds. Corrected using SDSS data. \\
\hline \multirow[t]{2}{*}{ RX1740 } & Field with high density of stars. \\
\hline & Bright star at $\left(265.30723^{\circ}, 35.73833^{\circ}\right)$ and $\left(265.41056^{\circ}, 35.36699^{\circ}\right)$ \\
\hline \multicolumn{2}{|l|}{ Z1261 } \\
\hline Z2844 & Very bright spike in the border of one chip $\left(150.98105^{\circ}, 32.64584^{\circ}\right)$ \\
\hline Z8338 & Field with high density of stars \\
\hline Z8852 & Very bright star at $\left(347.52789^{\circ}, 7.36402^{\circ}\right)$ \\
\hline
\end{tabular}

